\title{
Photoinactivation of catalase sensitizes Candida albicans and Candida auris to ROS-producing agents and immune cells
}

Pu-Ting Dong ${ }^{1,2, \#, ~ Y u e w e i ~ Z h a n ~}{ }^{1,2, \#}$, Sebastian Jusuf ${ }^{1,2, \#}$, Jie Hui ${ }^{1,2, \#}$, Zeina Dagher ${ }^{3,4}$, Michael K. Mansour ${ }^{3,4,}$, Ji-Xin Cheng ${ }^{1,2, *}$

${ }^{1}$ Department of Biomedical Engineering, Boston University, Boston, MA 02215, USA.

${ }^{2}$ Photonics Center, Boston University, Boston, MA 02215, USA.

${ }^{3}$ Division of Infectious Diseases, Massachusetts General Hospital, Boston, MA 02114, USA.

${ }^{4}$ Harvard Medical School, Boston, MA 02115, USA.

${ }^{\star}$ Corresponding authors. Emails: jxcheng@bu.edu and mkmansour@partners.org

\#These authors contributed equally: Pu-Ting Dong, Yuewei Zhan, Sebastian Jusuf, Jie Hui. 


\begin{abstract}
Nearly all organisms found in nature have evolved and developed their own specific strategies to cope with reactive oxygen species (ROS). Catalase, a heme-containing tetramer protein expressed in a broad range of aerobic fungi, has been utilized as an essential enzymatic ROS detoxifying mechanism, and shows remarkable efficiency in degrading hydrogen peroxide $\left(\mathrm{H}_{2} \mathrm{O}_{2}\right)$ for fungal cell survival and host invasion. Here, we demonstrate that catalase inactivation with blue light renders fungal cells highly susceptible to ROS attack, thus resembling a 'strength-toweakness optical switch'. To unveil catalase as the underlying molecular target of blue light and its inactivation mechanism, we systematically compared wild-type Candida albicans to a catalasedeficient mutant strain for susceptibility to ROS in the absence/presence of $410 \mathrm{~nm}$ treatment. Upon testing on a wide range of fungal species and strains, we found that intracellular catalase could be effectively and universally inactivated by $410 \mathrm{~nm}$ blue light. We find that the photoinactivation of catalase in combination with ROS-generating agents is highly effective and potent in achieving full eradication of multiple fungal species and strains, including multiple clinical strains of Candida auris, the causative agent of the global fungal epidemic. In addition, photoinactivation of catalase is shown to facilitate macrophage killing of intracellular Candida albicans. The antifungal efficacy of catalase photoinactivation is further validated using a Candida albicans-induced mouse model of skin abrasion. Taken together, our findings offer a novel catalase-photoinactivation approach to address multidrug-resistant Candida infections.
\end{abstract}




\section{Introduction}

The frequency of invasive fungal infections in immunocompromised patients has been consistently increasing over the past few decades (1). Candida species are the most common cause of human fungal infections (2), such as oropharyngeal, cutaneous candidiasis and mucosal or deep-seated organ infections (3). Invasive Candida infections remain a significant cause of morbidity and mortality among immunocompromised patients, partly due to the growing spread of antifungal resistance (4). The growing emergence of multidrug-resistant Candida species poses an alarming trend worldwide (5). Especially of concern is Candida auris ( $C$. auris) which has resulted in multiple worldwide healthcare outbreaks with record-breaking mortality rate due to its multi-drug or even pan-drug resistance nature and biofilm formation (6, 7). Ongoing COVID-19 pandemic has further accelerated $C$. auris outbreaks (8). Life-threatening invasive mycosis has been recorded to accompany COVID-19 patients due to impaired immune responses, forcing higher than normal usage of antifungal agents (9). At a higher level, the long-term imprudent usage of antifungal agents has been shown to accelerate the resistance of anti-fungal development (need a reference here). Confronted with this severe situation, novel therapeutic approaches are highly desired.

Antimicrobial blue light, especially in the 400-430 nm range, has drawn increasing attention in recent years as a non-drug approach to treat wide-ranging bacterial $(10,11)$ and fungal infections $(12,13)$. The antifungal efficacy of blue light has been studied by many research groups worldwide. Blue light at $415 \mathrm{~nm}$ has demonstrated the capability to inactivate $C$. albicans both in vitro and in vivo mouse burn infection models, and the susceptibility of $C$. albicans to blue light-induced inactivation did not change even after the $10^{\text {th }}$ passage in the presence of blue light exposure suggesting the unlikelihood of fungi developing photoresistance (14). Gupta et al. achieved 4.52$\log _{10}$ reduction of $C$. albicans after delivering $332.1 \mathrm{~J} / \mathrm{cm}^{2}$ of $405 \mathrm{~nm}$ irradiance (15). Rosa et al. reported a $2.3-\log _{10}$ reduction of $C$. albicans in a biofilm setting under the treatment of $455 \mathrm{~nm}$ exposure with a dose of $45.16 \mathrm{~J} / \mathrm{cm}^{2}$ (16). Raquel et al. further demonstrated significant antimicrobial effect of $405 \mathrm{~nm}$ on $C$. albicans both in monomicrobial and polymicrobial biofilm schemes (17). Blue light was also utilized to combine with other agents to eliminate $C$. albicans. Leon at al. showed that quinine chloride could enhance the fungicidal effects of blue light $405 \mathrm{~nm}$ on the elimination of $C$. albicans both in vitro and in vivo. Besides $C$. albicans, blue light can also inhibit the growth of microconidia of molds, such as Trichophyton rubrum and Trichophyton mentagrophytes (18). Studies also demonstrated that $405 \mathrm{~nm}$ light has detrimental effect on additional yeasts such as Sacchromyces cerevisiae and obligate molds including Aspergillus niger (19). Noteworthy, no significant loss of viability on human keratinocytes was observed after 216 $\mathrm{J} / \mathrm{cm}^{2}$ blue light treatment (17). Collectively, these studies demonstrate that blue light works effectively against major pathogenic fungal species without developing resistance and no significant cytotoxicity on host cells, thus emerging as a drug-free alternative approach to combat fungal infections.

However, the underlying antimicrobial mechanisms of blue light has stayed elusive for decades. A widely believed hypothesis is that metal-free porphyrin (in the $405-420 \mathrm{~nm}$ window) or riboflavin (in the 450-470 $\mathrm{nm}$ window) are endogenous molecular targets (12). Bacterial/fungal 
inactivation are eradicated by the reactive oxygen species (ROS) produced through photodynamic reaction between these molecules and blue light (20). However, this perspective remains controversial. First, it has been reported that the total amount of coprotoporphyrins inside the microbes was not a contributing factor to the antimicrobial efficacy of blue light (21). Second, the intracellular concentration of porphyrins or riboflavin is as low as $2-4 \times 10^{-3} \mathrm{mg} / \mathrm{ml}(22)$. Given the situation that research of antimicrobial blue light on fungi stays understudied and the working mechanism of antimicrobial blue light has yet to be clarified, other possible molecular targets are assumed to exist.

Here, using the wild type $C$. albicans SC5314 along with a catalase-deficient $C$. albicans mutant, we demonstrate that catalase is a major molecular target of antimicrobial blue light (400$420 \mathrm{~nm}$ window). Catalase from wide-ranging fungal species could be effectively inactivated by $410 \mathrm{~nm}$ blue light. Subsequently, photoinactivation of catalase renders fungal cells highly susceptible to non-fungicidal low-concentration hydrogen peroxide $\left(\mathrm{H}_{2} \mathrm{O}_{2}\right)$ and ROS-producing antifungal drugs. Moreover, the synergy between photoinactivation of catalase and $\mathrm{H}_{2} \mathrm{O}_{2}$ can enable total eradication of multiple notorious drug-resistant $C$. auris isolates whereas either alone has limited efficacy. Furthermore, photoinactivation of catalase substantially boosts macrophages activity against $C$. albicans indicated by shorter hyphae length and higher eradication percent of C. albicans were observed in the case of $410 \mathrm{~nm}$ involved group. Finally, we validated the synergistic effect between photoinactivation of catalase and $\mathrm{H}_{2} \mathrm{O}_{2}$ in a C. albicans-induced mouse skin abrasion model without noticeable skin damage. Collectively, our findings provide a novel catalase-targeting strategy to treat multi-drug resistant fungal infections.

\section{Results}

\section{Catalase inside fungal cells can be inactivated by blue light irradiance}

As shown in Figure 1a, catalase (from bovine liver) revealed by PyMOL has a tetramer structure, with porphyrin rings hiding inside its hydrophobic pocket. It was reported as early as in 1965 that bovine liver catalase can be inactivated by visible light exposure (23). Nonetheless, photoinactivation of catalase remained underexplored over the decades. To better understand how photons inactivate catalase, especially catalase inside fungal cells, we first quantified the remaining catalase percentage from bovine liver catalase $(2.5 \mathrm{U} / \mathrm{ml})$ after blue light exposure under various wavelengths using an Amplex red catalase kit. And we found that blue light, especially in the 400-420 nm window, inactivates catalase by $70 \%$ compared to control (Figure 1b). $410 \mathrm{~nm}$ shows the best capability to inactivate catalase, which might be due to the fact that the optimal absorption peak of catalase is around $410 \mathrm{~nm}$. In the following experiments, we used continuouswave (CW) $410 \mathrm{~nm}$ LED as the light source to inactivate catalase.

To query whether fungal catalase can be inactivated by blue light in the same way as liver catalase, we tested the remaining catalase percentage inside the fungal cells after $410 \mathrm{~nm}$ exposure. Prior to that, we investigated the catalase expression level for fungal cells at different metabolic phases. It was reported that stationary-phase microbes usually have higher amount of sigma factor $\sigma^{S}$, the key protein responsible for survival and improved resistance under stressful conditions 
(24). Increased expression of $\sigma^{S}$ leads to the relatively higher amount of catalase in the stationaryphase microbes (24). As shown in Figure 1c-d, we indeed found that stationary-phase Candida spp. have more catalase compared to log-phase. Of note, catalase in log-and stationary-phase $C$. albicans could be consistently inactivated by $410 \mathrm{~nm}$ exposure. Moreover, this inactivation behavior exists in many other fungal strains apart from C. albicans (Figure 1e). In short, we found that catalase inside fungal cells can be effectively inactivated by $410 \mathrm{~nm}$ exposure regardless catalase expression level.
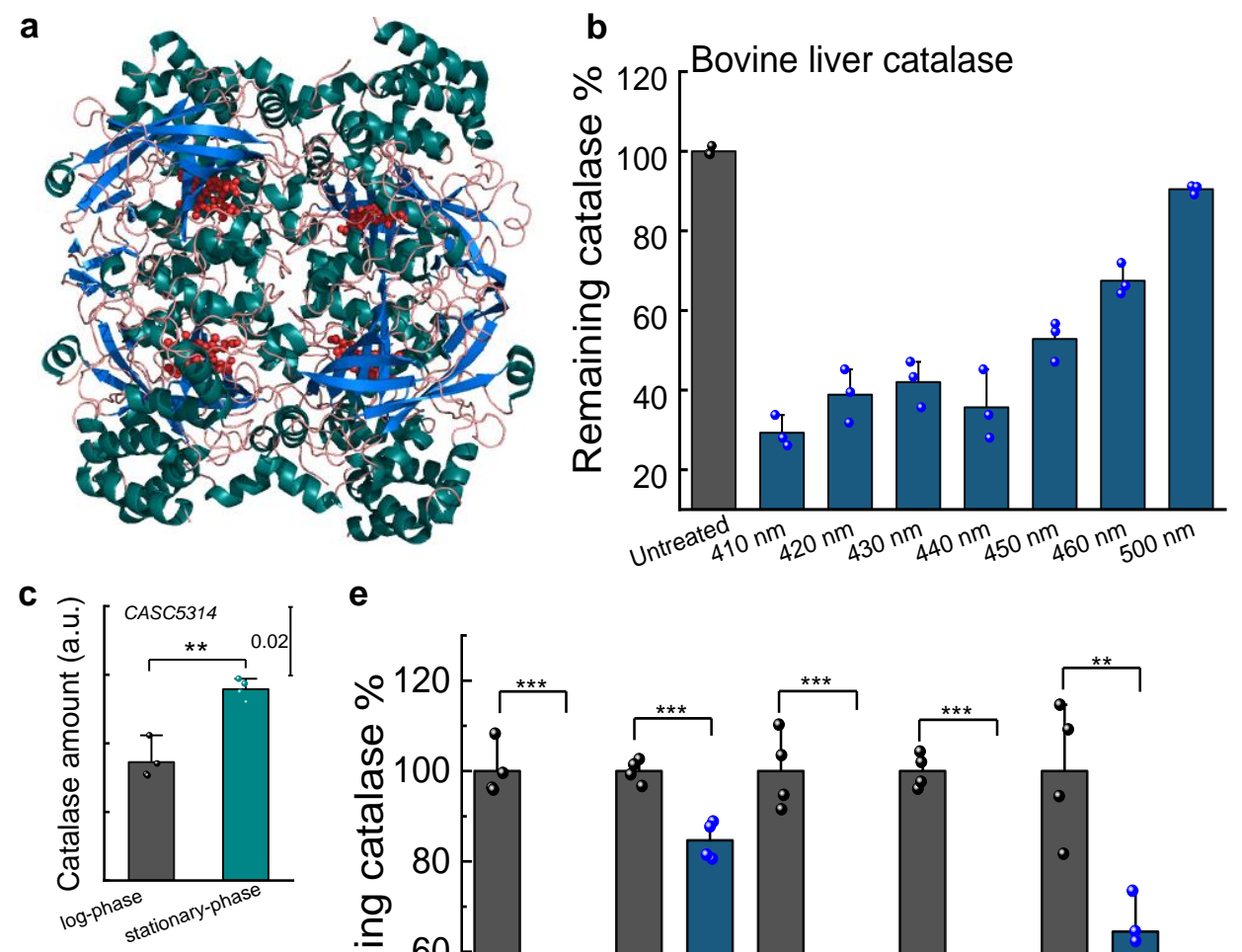

e

d
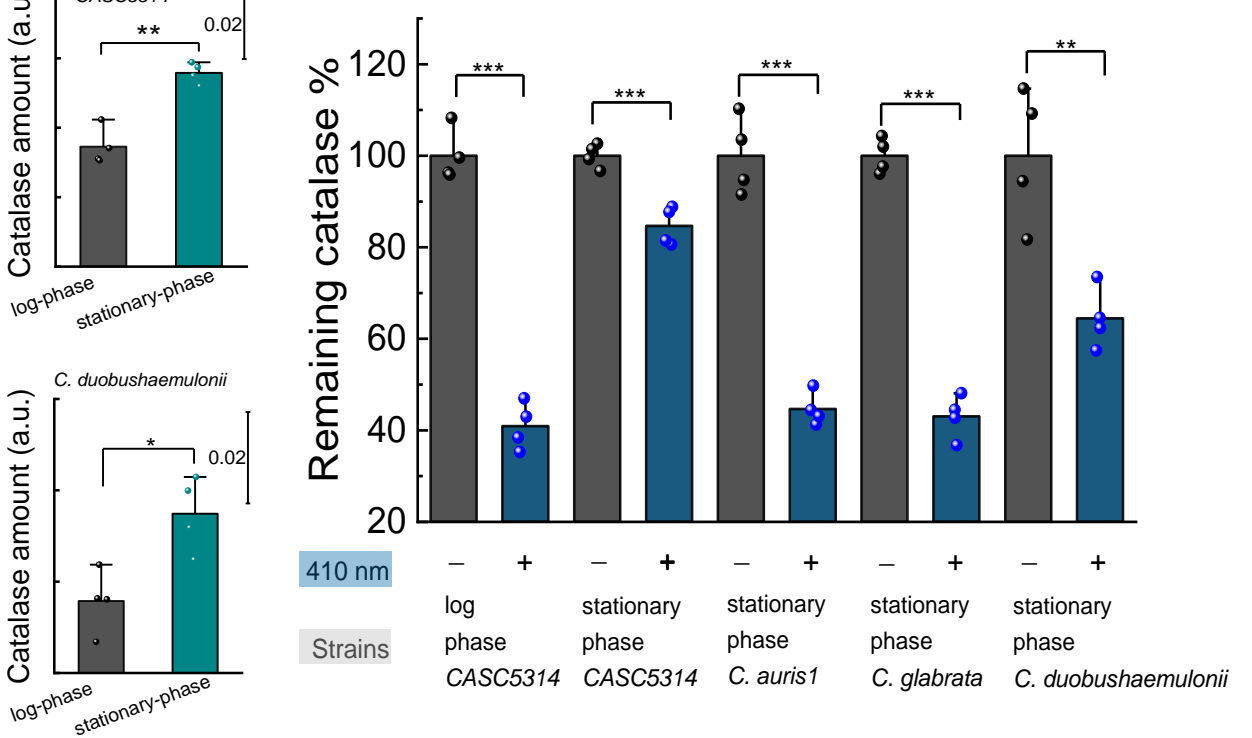

Figure 1. Catalase from bovine liver and fungi cells can be inactivated by blue light. a. Structure of bovine liver catalase revealed by PyMOL simulation. PDB ID: 1TGU. b. Quantification of remaining catalase percentage of bovine liver catalase $(2.5 \mathrm{U} / \mathrm{ml})$ by Amplex Red catalase assay under different treatment schemes. $\mathrm{N}=3$. Dose: $15 \mathrm{~J} / \mathrm{cm}^{2}$. c-d. Comparison of catalase amount between log- and stationaryphase C. albicans SC5314 (c) and Candida duobushaemulonii (d). e. Quantification of remaining catalase percentage in various fungal strains with and without $410 \mathrm{~nm}$ blue light. Dose: $30 \mathrm{~J} / \mathrm{cm}^{2}$. Data: Mean+SD from at least three replicates. Student unpaired $t$-test. ${ }^{*}: p<0.05,{ }^{* *}: p<0.01 ;{ }^{* *}: p<0.001$. 


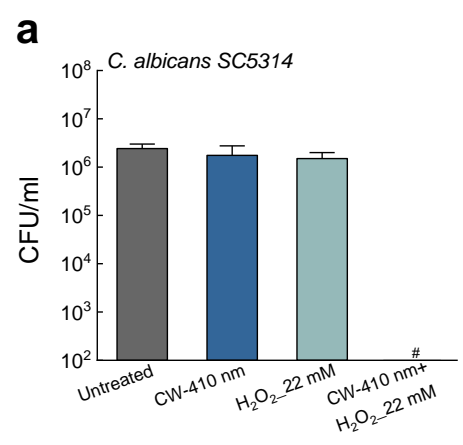

b
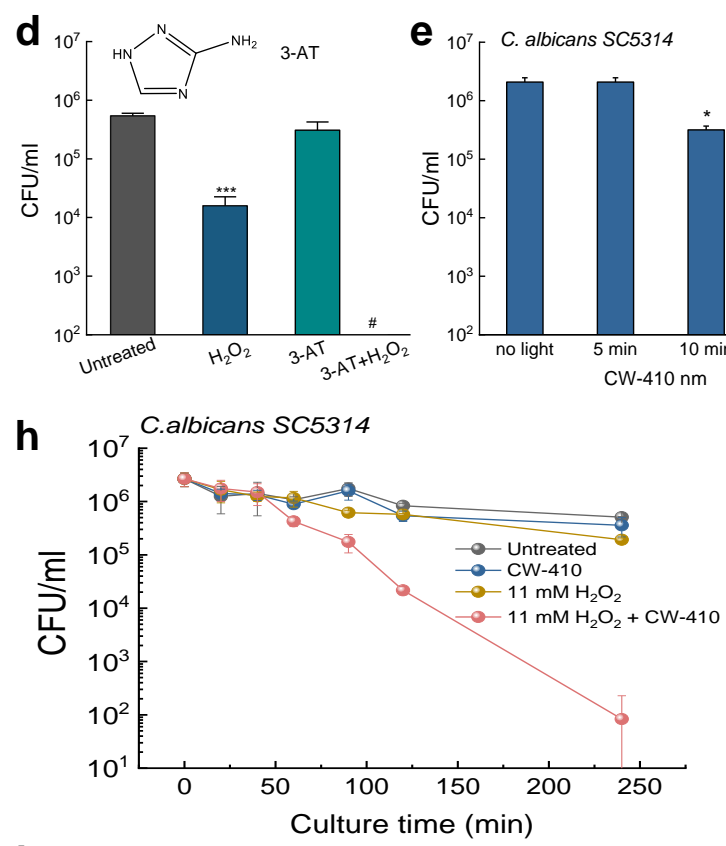

j $\mathrm{H}_{2} \mathrm{O}_{2}$

C.albicans SC5314
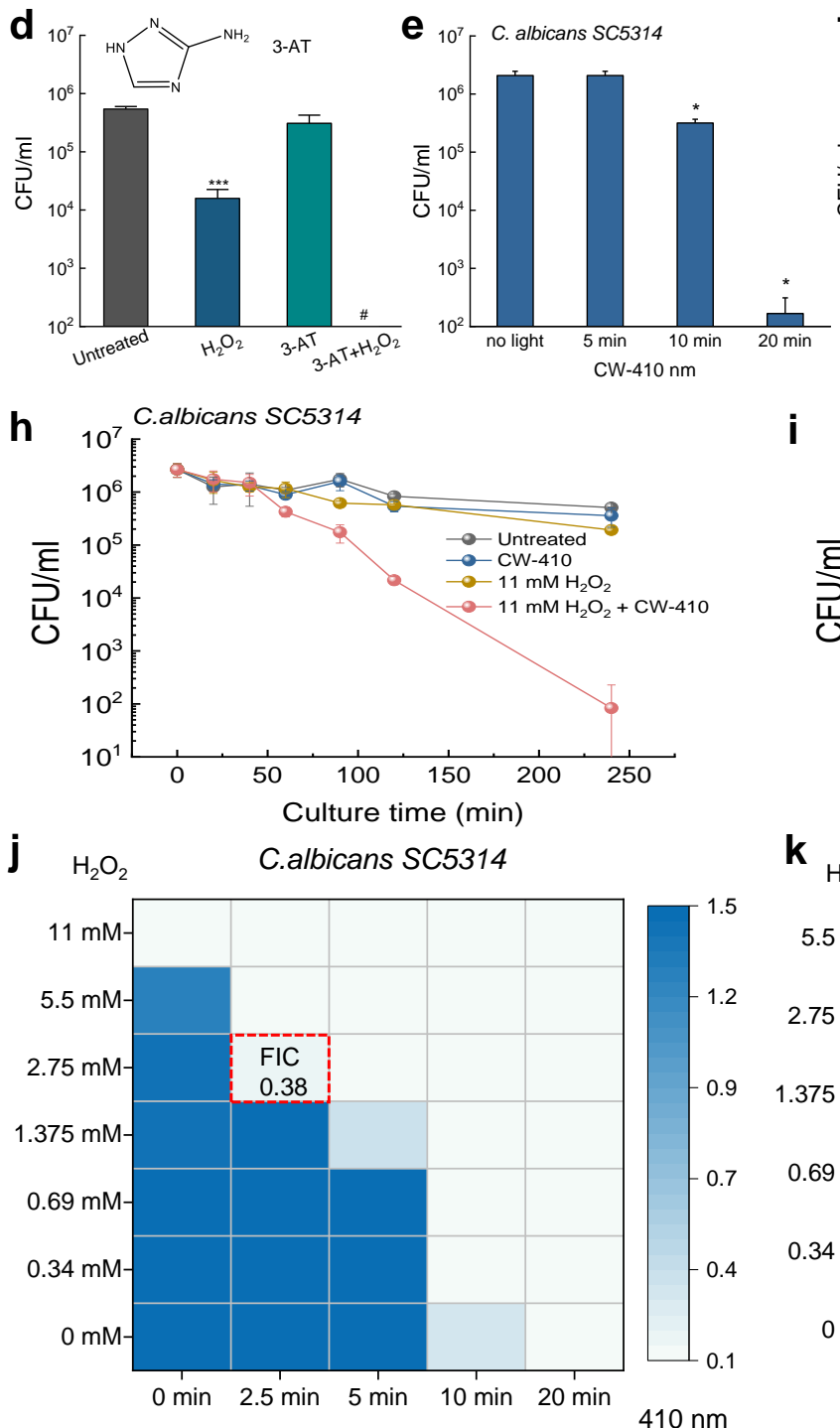

\section{i}

k

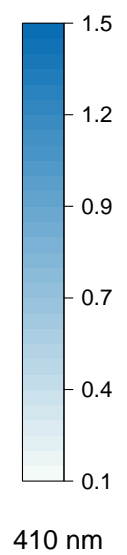

$+\mathrm{H}_{2} \mathrm{O}_{2}$
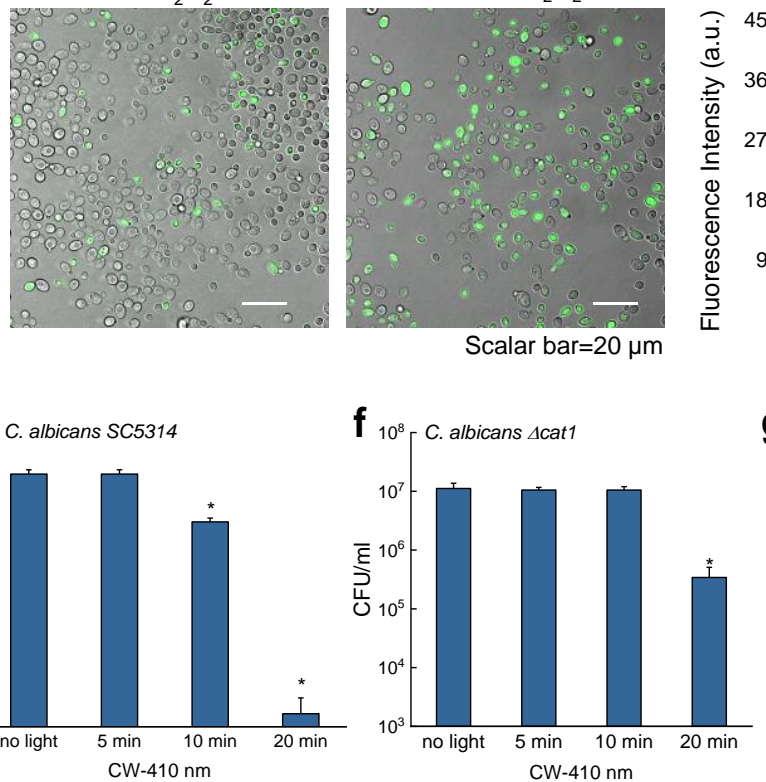

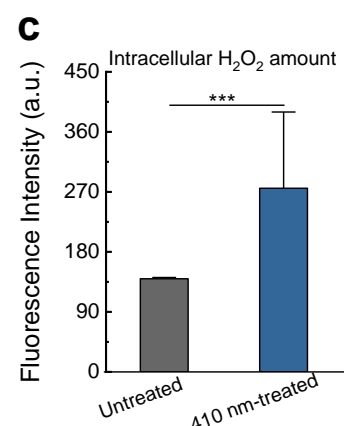

g

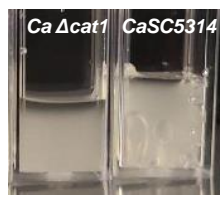

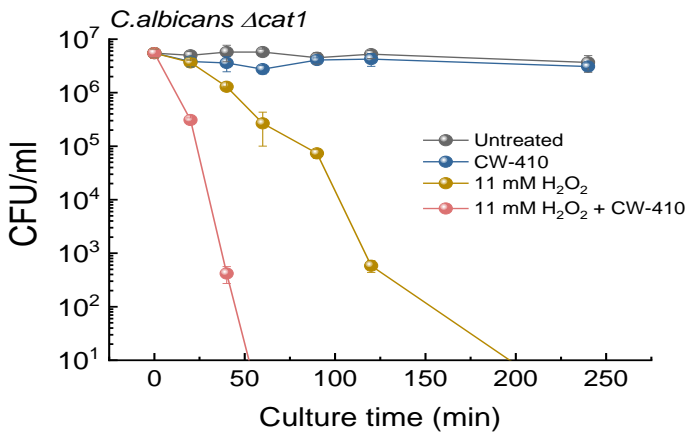

Figure 2. Photoinactivation of catalase sensitizes $C$. albicans to $\mathrm{H}_{2} \mathrm{O}_{2}$ and catalase is identified as a primary molecular target of blue light. a. Colony-forming unit (CFU/ml) of C. albicans SC5314 under different treatment schemes. b. Confocal imaging of intracellular $\mathrm{H}_{2} \mathrm{O}_{2}$ with/without $410 \mathrm{~nm}$ exposure. Scalar bar $=20 \mu \mathrm{m} . \mathrm{H}_{2} \mathrm{O}_{2}: 22 \mathrm{mM}$, images were taken after 30-min incubation and washing step. c. Quantitation of intracellular $\mathrm{H}_{2} \mathrm{O}_{2}$ via fluorescence intensity from images acquired in b. d. CFU/ml of $C$. albicans SC5314 in the presence of 3-AT. Significant difference from the untreated group. 3-AT: $50 \mathrm{mM}$, 
4-hour incubation. e-f. CFU/ml of C. albicans SC5314 and its catalase-deficient mutant C. albicans $\Delta$ cat1 under $410 \mathrm{~nm}$ exposure with various doses. g. Pictures showing bubble formation phenomena of C. albicans $\Delta$ cat1 and C. albicans SC5314 in the presence of $3 \% \mathrm{H}_{2} \mathrm{O}_{2}$. h-i. Time-killing curves of C. albicans SC5314 (h) and C. albicans $\Delta$ catl (i) under different treatment schemes. $\mathbf{j}$-k. Checkerboard broth dilution assay showing the combinatorial behavior between blue light and $\mathrm{H}_{2} \mathrm{O}_{2}$ against $C$. albicans SC5314 and $C$. albicans $\Delta$ cat 1 . Data: Mean+SD. N=3. Student unpaired $t$-test. ${ }^{*}: p<0.05,{ }^{* *}: p<0.01 ;{ }^{* * *}: p<0.001$. Pound sign (\#) means CFU results are below the detection limit.

\section{Photoinactivation of catalase sensitizes $C$. albicans to $\mathrm{H}_{2} \mathrm{O}_{2}$}

Having established that fungal catalase can be efficiently inactivated by $410 \mathrm{~nm}$ blue light, we then queried whether this attenuation effect sensitizes $C$. albicans to $\mathrm{H}_{2} \mathrm{O}_{2}$ considering the major function of catalase. A colony-forming unit (CFU/ml) assay was conducted by combining $410 \mathrm{~nm}$ with $\mathrm{H}_{2} \mathrm{O}_{2}$ at a non-fungicidal concentration $(22 \mathrm{mM})$. As shown in Figure $2 \mathbf{a}$, photoinactivation of catalase significantly sensitizes wild-type $C$. albicans SC5314 to $\mathrm{H}_{2} \mathrm{O}_{2}$ by around six orders of magnitude, whereas $\mathrm{H}_{2} \mathrm{O}_{2}$ or blue light alone didn't have apparent killing effect. This result indicates a synergistic killing effect between photoinactivation of catalase and $\mathrm{H}_{2} \mathrm{O}_{2}$. To understand whether this augmented killing was due to intracellular accumulation of $\mathrm{H}_{2} \mathrm{O}_{2}$ after catalase inactivation, we utilized an established kit using $\mathrm{H}_{2} \mathrm{O}_{2}$-sensitive fluorophore to quantify intracellular $\mathrm{H}_{2} \mathrm{O}_{2}$ in $C$. albicans cells treated with $\mathrm{H}_{2} \mathrm{O}_{2}$ or $410 \mathrm{~nm}$ plus $\mathrm{H}_{2} \mathrm{O}_{2}$ through a confocal laser scanning microscope (CLSM). As shown in Figure 2b-c, fluorescent intensity in the $410 \mathrm{~nm}$ plus $\mathrm{H}_{2} \mathrm{O}_{2}$ treated single $C$. albicans $\mathrm{SC} 5314$ was significantly higher than cells treated by $\mathrm{H}_{2} \mathrm{O}_{2}$ alone, suggesting that photoinactivation of catalase boosts intracellular $\mathrm{H}_{2} \mathrm{O}_{2}$ accumulation.

To investigate whether catalase is the primary target for blue light exposure, we conducted the following experiments. First, a catalase inhibitor, 3-Amino-1,2,4-triazole (3-AT) (25), was utilized to chemically inhibit catalase activity within C. albicans, and then the susceptibility to $\mathrm{H}_{2} \mathrm{O}_{2}$ was recorded. As shown in Figure 2d, total eradication was obtained when adding $\mathrm{H}_{2} \mathrm{O}_{2}$ to 3-AT treated $C$. albicans SC5314 whereas 3-AT alone only exerts limited killing efficiency, suggesting the pivotal role that catalase plays in neutralizing $\mathrm{H}_{2} \mathrm{O}_{2}$. Second, we exploited a catalase-deficient C. albicans strain, $C$. albicans $\Delta$ cat1, to further evaluate the function of catalase underlying the antimicrobial effect of blue light. $C$. albicans $\Delta$ cat 1 demonstrates significantly lower susceptibility to $410 \mathrm{~nm}$ blue light compared to its the wild type (Figure 2e-f), indicating catalase-mediated sensitivity to $410 \mathrm{~nm}$ blue light.

Next we examined the time-kill CFU assay of wild type C. albicans SC5314 and catalasedeficient $C$. albicans $\Delta$ cat 1 under different treatment schemes. $C$. albicans $\Delta$ cat 1 did not produce prototypical oxygen bubbles as the wild type C. albicans $S C 5314$ did in the presence of $\mathrm{H}_{2} \mathrm{O}_{2}$ (Figure 2g), confirming the major role of catalase in $\mathrm{H}_{2} \mathrm{O}_{2}$ neutralization. As shown in Figure 2h, four hours after treatments, both $\mathrm{H}_{2} \mathrm{O}_{2}$ and $410 \mathrm{~nm}$ blue light alone didn't exert significant fungicidal effects, whereas $\mathrm{H}_{2} \mathrm{O}_{2}$ plus $410 \mathrm{~nm}$ blue light-treated group had around a $5-\log _{10}$ reduction of wild-type $C$. albicans SC5314. These data suggest that there is an ample synergy between photoinactivation of catalase and $\mathrm{H}_{2} \mathrm{O}_{2}$ against $C$. albicans. Of note, when applying the same $\mathrm{H}_{2} \mathrm{O}_{2}$ with concentration used to test wild type $C$. albicans $\mathrm{SC} 5314, \mathrm{H}_{2} \mathrm{O}_{2}$ alone achieved 
more than $5-\log _{10}$ reduction of $C$. albicans $\Delta$ catl (Figure 2i, supplementary Figure 1a). Moreover, C. albicans $\Delta$ cat 1 exhibited similar susceptibility to $\mathrm{H}_{2} \mathrm{O}_{2}$ killing compared to wild type C. albicans SC5314 exposed to $410 \mathrm{~nm}$ plus $\mathrm{H}_{2} \mathrm{O}_{2}$ in a time-kill assay, corroborating that catalase is a key target of $410 \mathrm{~nm}$ light. Noteworthy, enhanced $\mathrm{H}_{2} \mathrm{O}_{2}$ killing of C. albicans $\Delta$ cat1 after 410 $\mathrm{nm}$ exposure was observed (Figure 2i, supplementary Figure 1b), hinting those additional molecular targets other than catalase might exist and be responsive to $410 \mathrm{~nm}$ exposure.

To further confirm the synergistic effect between photoinactivation of catalase and $\mathrm{H}_{2} \mathrm{O}_{2}$, we conducted a checkerboard broth dilution assay to derive a fractional inhibitory concentration index (FICI) (26). FICI was calculated through the following equation:

$$
F I C I=F I C_{A}+F I C_{B}=\frac{M I C_{A}^{C o m b}}{M I C_{A}^{\text {alone }}}+\frac{M I C_{B}^{C o m b}}{M I C_{B}^{\text {alone }}}
$$

, where $M I C_{A}^{\text {alone }}$ and $M I C_{A}^{\text {alone }}$ are the minimal inhibitory concentrations (MICs) of the drugs A and $\mathrm{B}$ when functioning alone, and $M I C_{A}^{C o m b}$ and $M I C_{B}^{C o m b}$ are the MICs of drugs $\mathrm{A}$ and $\mathrm{B}$ in combination, respectively. A synergy between two agents was defined with FICI $\leq 0.5$ (27), and 'no interaction' with a FICI in the range of 0.5-4 (27). The MICs of $410 \mathrm{~nm}$ against $C$. albicans SC5314 and C. albicans $\Delta$ cat1 were $282 \mathrm{~J} / \mathrm{cm}^{2}$ and $564 \mathrm{~J} / \mathrm{cm}^{2}$, respectively (supplementary Figure 2a, c). And MICs of $\mathrm{H}_{2} \mathrm{O}_{2}$ were $11 \mathrm{mM}$ and $2.75 \mathrm{mM}$, respectively (supplementary Figure 2b, d). Once combining $410 \mathrm{~nm}$ and $\mathrm{H}_{2} \mathrm{O}_{2}$ together, $\mathrm{MIC}$ of $\mathrm{H}_{2} \mathrm{O}_{2}$ against wild type $C$. albicans SC5314 was around $2.75 \mathrm{mM}$ (Figure $\mathbf{2 j}$ ), and $1.375 \mathrm{mM}$ against $C$. albicans $\Delta$ cat 1 (Figure $\mathbf{2 k}$ ). FICI from both strains could be calculated from the above equation. Then we obtained a FICI of 0.38 in the case of wild type $C$. albicans SC5314, and a FICI of 0.75 for $C$. albicans $\Delta$ cat 1 . These results indicate that there is an effective synergy between photoinactivation of catalase and $\mathrm{H}_{2} \mathrm{O}_{2}$ in eradicating $C$. albicans.

\section{Photoinactivation of catalase enhances ROS-generating agents to inhibit the proliferation of wide-ranging fungal cells}

After demonstrating the synergy between photoinactivation of catalase with non-fungicidal low-concentration $\mathrm{H}_{2} \mathrm{O}_{2}$ against $C$. albicans, we next query whether this synergy still holds effective against other fungal species. To have a high-throughput analysis, we adopted a PrestoBlue viability assay (28) to investigate the time-course proliferation of wide-ranging fungal species under different treatment schemes (supplementary Figures 3-4).

As shown in Figure 3a, photoinactivation of catalase consistently enhances the fungistatic effect of $\mathrm{H}_{2} \mathrm{O}_{2}$ against both $\log$ - and stationary-phase $C$. albicans $\mathrm{SC} 5314$, and $C$. albicans $\mathrm{C} 13$, C. albicans $\mathrm{C} 14$ as indicated by nearly $0 \%$ proliferation rate in the combination-treated group. Besides $C$. albicans, we also investigated whether this enhancement works for non-albicans Candida species. Candida glabrata (C. glabrata), the commensal saprophyte, caused increasing incidence and prevalence of mucosal and systemic infections due to the widespread use of immunosuppressive and antimycotic therapy (29). Candida parapsilosis ( $C$. parapsilosis), the second most commonly isolated Candida species from blood cultures, could also cause invasive 
C. parapsilosis infections in both AIDS patients and surgical patients (30). Candida lusitaniae (C. lusitaniae), fungal pathogen capable of developing both acute and long-term infections, can rapidly develop resistance (31) to multiple antifungals and lead to the breakouts of multidrugresistant fungal infections (32). Candida haemulonii (C. haemulonii), the opportunistic

a
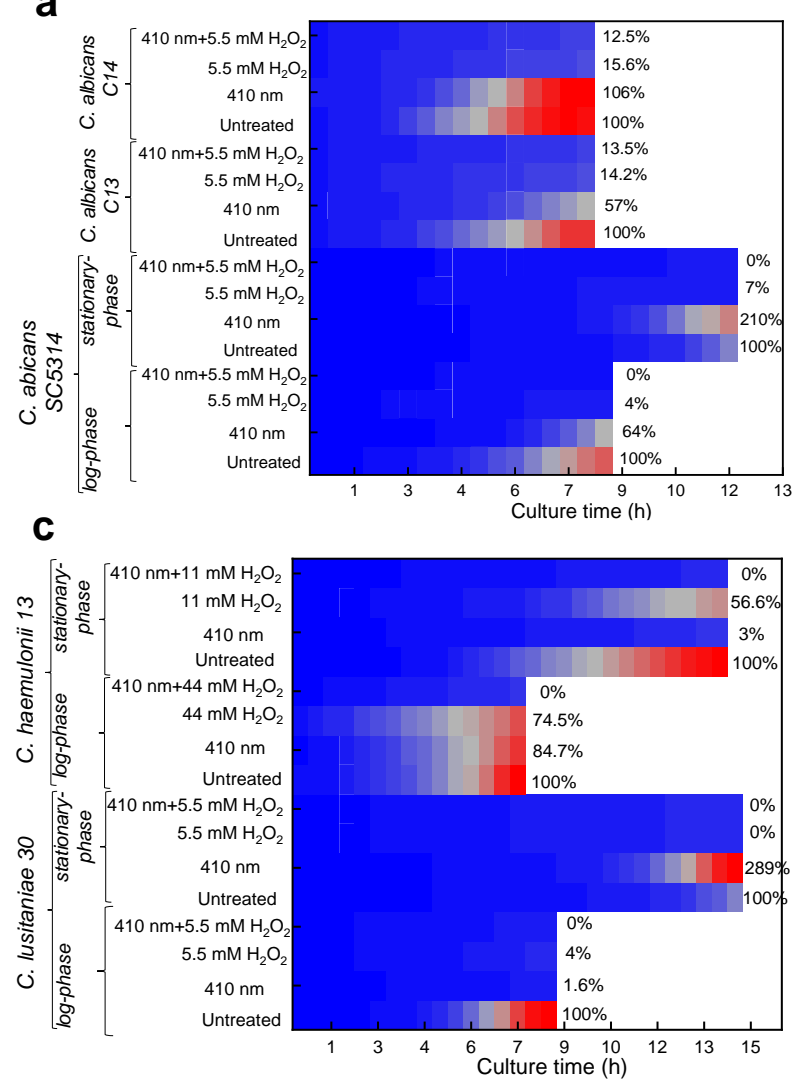

e

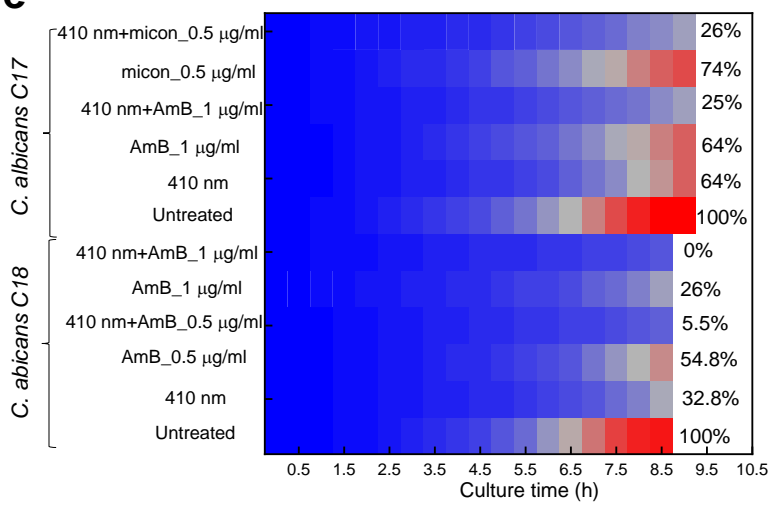

b

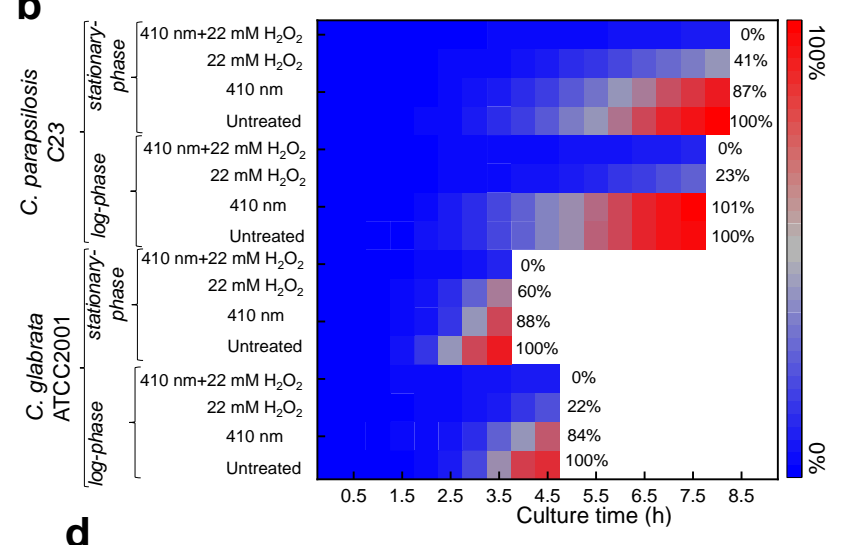

d

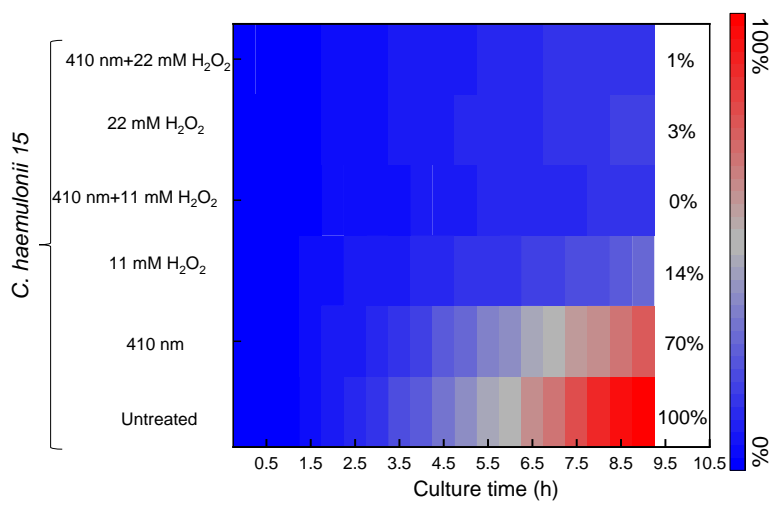

f

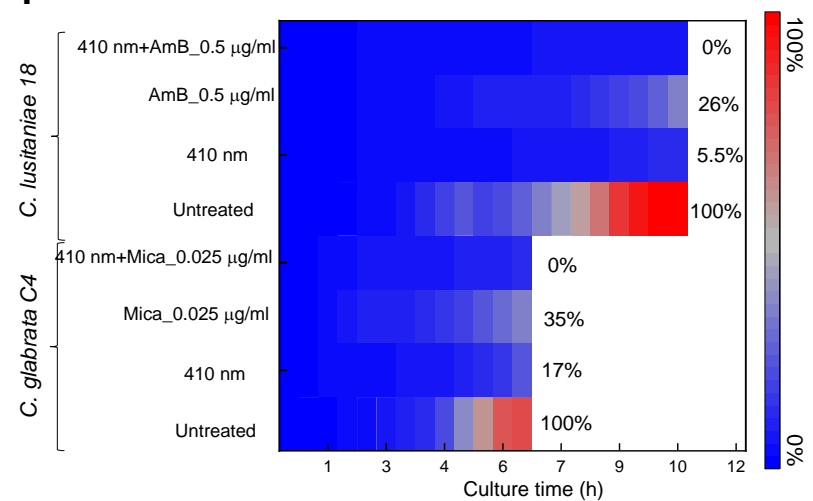

Figure 3. Photoinactivation of catalase enhances ROS-generating agents to inhibit the proliferation of wide-ranging fungal cells. Data were acquired through PrestoBlue proliferation assay and were presented as mean from three replicates. Blue light: $410 \mathrm{~nm}, 30 \mathrm{~J} / \mathrm{cm}^{2}$. Abbreviations: AmB (amphotericin B), micon (miconazole), mica (micafungin).

fungal pathogen associated with bloodstream infections, has emerged as a multi-drug resistant yeast for deep-seated soft tissue and bone infections in diabetic patients (33). Thus, we further 
wondered whether photoinactivation of catalase enhances the antifungal efficacy of $\mathrm{H}_{2} \mathrm{O}_{2}$ against these fungal species.

Noteworthy, complete inhibition of proliferation ( $0 \%$ growth compared to the untreated group) was obtained from all the above mentioned fungal species in the $410 \mathrm{~nm}$ plus $\mathrm{H}_{2} \mathrm{O}_{2}$ treated group (Figure 3, b-d; supplementary Figure 3), both in the form of log- and stationary-phase. For example, in the case of stationary-phase C. glabrata ATCC2001 (Figure 3b), $410 \mathrm{~nm}$ exposure $\left(30 \mathrm{~J} / \mathrm{cm}^{2}\right)$ inhibited around $12 \%$ of the original fungal cells to proliferate. At a concentration of $22 \mathrm{mM}, \mathrm{H}_{2} \mathrm{O}_{2}$ alone prohibited the growth of around $40 \%$ of the original fungal cells. Once combining these two treatments together, total growth inhibition was obtained. These findings consolidated the synergy between photoinactivation of catalase and $\mathrm{H}_{2} \mathrm{O}_{2}$ against those catalasepositive fungal pathogens, which holds clinical potential for treating multidrug-resistant fungal infections.

To treat fungal infections in the clinic, currently there are limited classes of antifungals available, such as liposomal formulations of amphotericin B (representative of polyenes), miconazole (azole representative), echinocandin (antifungal which inhibits the cell wall synthesis). Interestingly, it was reported that all these antifungals are able to induce an intracellular ROS burst within fungal cells as part of their proposed antifungal mechanism (34-36). Considering the pivotal role of catalase in scavenging ROS (37) and the fact that fluconazole and amphotericin-B resistance are associated with increased expression of catalase and superoxide dismutase (38), we reasoned that photoinactivation of catalase could enhance the antifungal efficacy of these antimycotics against fungal cells. The same time-course PrestoBlue proliferation assay was applied for the evaluation when treating fungal cells with these antifungals at a sublethal concentration (supplementary Figure 4).

As shown in Figure 3e, in the case of $C$. albicans $C 17,410 \mathrm{~nm}$ blue light alone $\left(30 \mathrm{~J} / \mathrm{cm}^{2}\right)$ inhibited around $36 \%$ of original $C$. albicans to divide, amphotericin B (AmB, $1 \mu \mathrm{g} / \mathrm{ml})$ alone also gave out around $36 \%$ inhibition effects, whereas the administration of AmB after $410 \mathrm{~nm}$ blue light treatment drastically suppressed fungal growth by $75 \%$. Similar phenomenon was found in the combinational behavior between $410 \mathrm{~nm}$ treatment and miconazole (micon, $0.5 \mu \mathrm{g} / \mathrm{ml}$ ) against C. albicans (Figure 3e). Micafungin (mica, $0.025 \mu \mathrm{g} / \mathrm{ml}$ ) has demonstrated the augmented inhibition effect induced by photoinactivation of catalase against $C$. glabrata as well (Figure 3f). In combination with the time-course calibration curves with known number of fungal cells under the same conditions, we can derive the relatively proliferated fungal cell numbers (supplementary Figure 5-6). Through comparison between the proliferated fungal cell numbers under different treatment schemes, we can clearly observe the enhanced inhibition effect by $410 \mathrm{~nm}$ (supplementary Figure 6). Collectively, these data were in line with our hypothesis, that is, photoinactivation of catalase boosts the antimycotic efficacy of commonly used antifungal agents.

\section{Elimination of Candida auris by synergizing photoinactivation of catalase with $\mathrm{H}_{2} \mathrm{O}_{2}$}

Candida auris (C. auris), as an emerging and particularly notable Candida species, has been associated with nosocomial outbreaks on five continents since 2009 due to its innate resistance to 
multiple classes of antifungal drugs $(39,40)$. Its high morbidity and mortality have led to it being one of the major public health concerns worldwide. C. auris demonstrates a unique propensity to colonize and persist on various surfaces, contributing to multiple outbreaks in health care settings (41). C. auris has also been reported to cause bloodstream infections and urinary tract infections for patients with COVID-19 (8, 41), thus likely as a compounding factor in COVID-19 pandemic. Most of the clinical isolated $C$. auris strains exhibit resistance to triazoles (fluconazole) and other antifungal drugs (42). Confronted with this situation, it is imperative to have alternative approaches to both sterilize clinical settings and further treat $C$. auris-caused infections.

C. auris strains have been reported to be extremely resistant to hydrogen peroxide vapor sterilization (43), this triggered us to wonder whether this persistence is due to catalase. To investigate whether photoinactivation of catalase and $\mathrm{H}_{2} \mathrm{O}_{2}$ could efficiently kill $C$. auris, we conducted CFU assay of multiple $C$. auris clinical isolates acquired from antimicrobial resistance bank (AR-BANK) of the Centers for Disease Control and Prevention (CDC). Here C. auris 1, 2, 5, 6 represent AR-BANK\#0381, 0382, 0385, 0386, respectively. 

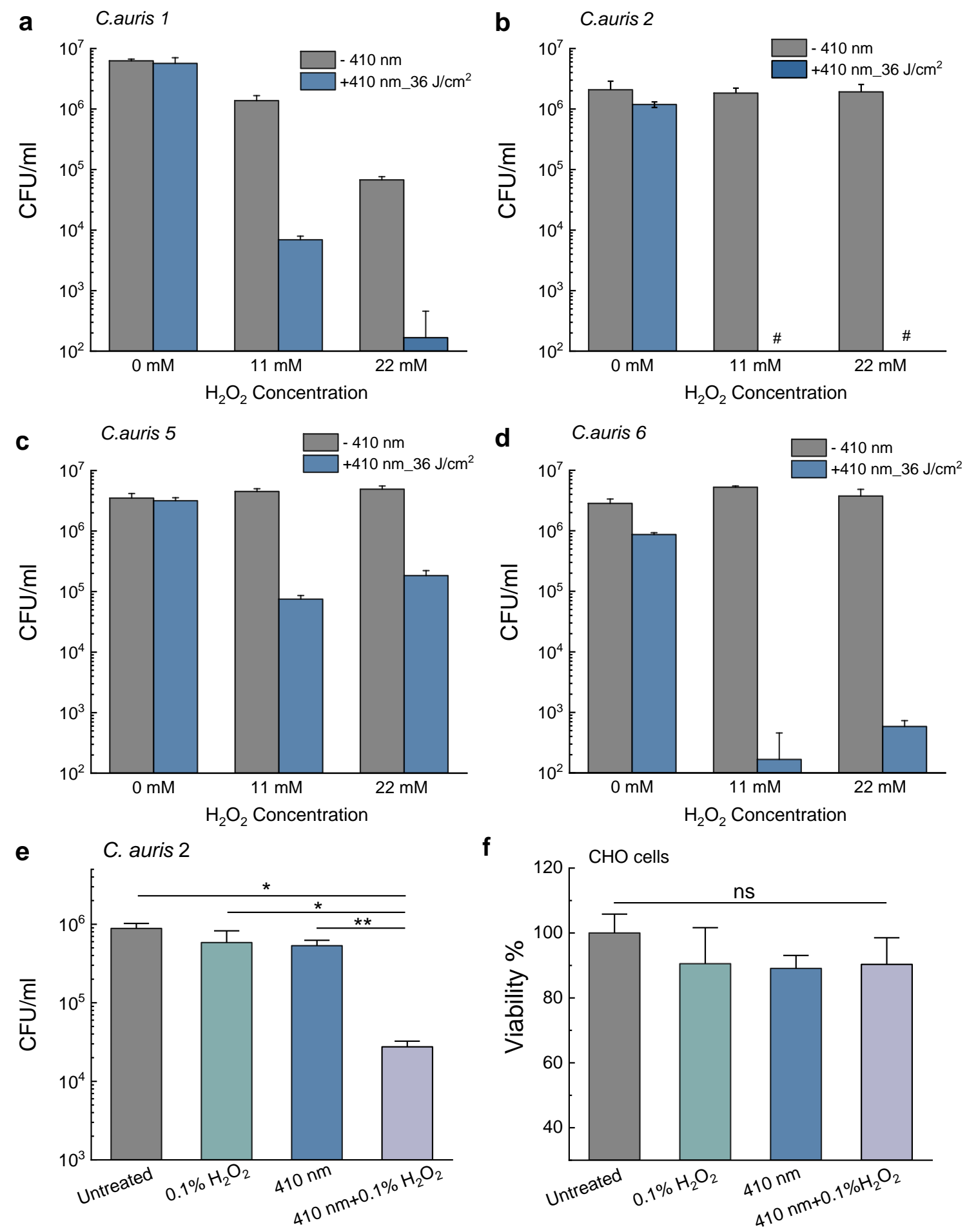

Figure 4. Elimination of various clinical Candida auris strains via synergy between photoinactivation of catalase with $\mathbf{H}_{2} \mathbf{O}_{2}$. CFU/ml of log-phase $C$. auris 1 (a), C. auris 2 (b), C. auris 5 (c), and C. auris 6 (d) under different treatment schemes. Data: Mean+SD. N=3. Pound sign (\#) means below the detection limit. $\mathrm{H}_{2} \mathrm{O}_{2}$-incubation time: 4 hours. $410 \mathrm{~nm}: 36 \mathrm{~J} / \mathrm{cm}^{2}$. (e). CFU/ml of $C$. auris 2 under $410 \mathrm{~nm}$ blue light followed by short-time incubation with $0.1 \% \mathrm{H}_{2} \mathrm{O}_{2}$ ( $30 \mathrm{mM}, 1$-min incubation). $410 \mathrm{~nm}$ blue light dose: 30 
$\mathrm{J} / \mathrm{cm}^{2}$. (f). Viability of CHO cells by MTT assay under the same treatment conditions as panel (e). Statistical analysis was achieved by student unpaired $t$-test. ${ }^{* *}: p<0.01 ;{ }^{*}: p<0.05$; ns: not significant.

Log-phase $C$. auris isolates were exposed to $410 \mathrm{~nm}$ blue light $\left(36 \mathrm{~J} / \mathrm{cm}^{2}\right)$ followed by subsequent administration of $\mathrm{H}_{2} \mathrm{O}_{2}$ (4-h incubation at $30^{\circ} \mathrm{C}, 11-22 \mathrm{mM}$ ), serial dilution and CFU enumeration. As shown in Figure $\mathbf{4 a}$, in the case of $C$. auris 1, photoinactivation of catalase renders this pathogen highly susceptible to $\mathrm{H}_{2} \mathrm{O}_{2}$ by around three orders of magnitude. Drastically, total eradication of $C$. auris 2 was obtained in the $410 \mathrm{~nm}$ plus $\mathrm{H}_{2} \mathrm{O}_{2}$ treated group (Figure 4b). Similar augmentation effect appeared with regard to $C$. auris 5 and $C$. auris 6 as well (Figure 4cd). These results underline the essential role of catalase for $C$. auris to defend external $\mathrm{H}_{2} \mathrm{O}_{2}$ attack. Furthermore, photoinactivation of catalase works synergistically with $\mathrm{H}_{2} \mathrm{O}_{2}$ against clinical $C$. auris.

It was reported that hydrogen peroxide vapor was utilized as clinical room disinfection in order to get rid of $C$. auris colonization (44), however the $C$. auris biofilm is difficult to eradicate explaining the concerning epidemiology of high relapse rates in clinical settings (43). To query the clinical potential of this synergistic therapy which requires shortened $\mathrm{H}_{2} \mathrm{O}_{2}$ treatment time, we then reduced both the $\mathrm{H}_{2} \mathrm{O}_{2}$ incubation time and $410 \mathrm{~nm}$ blue light treatment time and tested if such synergistic treatment remains effective against $C$. auris. We treated $410 \mathrm{~nm}$ blue lightexposed $C$. auris 2 with $\mathrm{H}_{2} \mathrm{O}_{2}$ at a higher concentration $(30 \mathrm{mM}, 0.1 \%$ ) for only 1 minute, then followed by $\mathrm{CFU}$ enumeration. As shown in Figure 4e, there was no significant difference between the untreated group and either blue light alone-treated group or $\mathrm{H}_{2} \mathrm{O}_{2}$ alone-treated group. However, around 99\% of fungal burden (Figure 4e) was reduced once combining these two treatments together, suggesting the consistent and effective synergy between photoinactivation of catalase and $\mathrm{H}_{2} \mathrm{O}_{2}$. Noteworthy, the same treatment schemes did not exert detrimental effects to a Chinese Hamster Ovary (CHO) cell line according to MTT assay (Figure 4f). Collectively, photoinactivation of catalase and $\mathrm{H}_{2} \mathrm{O}_{2}$ exhibit synergistic fungicidal effects against multiple clinical C. auris strains.

\section{Proliferation inhibition of wide-ranging $C$. auris strains by combining photoinactivation of catalase with ROS-producing agents}

Due to the prominent significance of $C$. auris-caused breakouts, we particularly investigated whether photoinactivation of catalase could sensitize wide-ranging $C$. auris strains to multiple ROS-producing agents $\left(\mathrm{H}_{2} \mathrm{O}_{2}\right.$, amphotericin $\mathrm{B}$, fluconazole) in a high-throughput approach, PrestoBlue proliferation assay was utilized again to investigate the fungistatic effects under various treatment schemes (supplementary Figure 7-11). Meanwhile, time-course PrestoBlue fluorescence intensity of untreated fungal cells with known CFU number were recorded as standard curves. In this way, we could derive the relatively proliferated fungal cell number from the treated groups based on the linear calibration curves (supplementary Figure 12-13).

As shown in Figure 5a-b, $410 \mathrm{~nm}$ blue light alone $\left(30 \mathrm{~J} / \mathrm{cm}^{2}\right)$ inhibits around the proliferation of $C$. auris 1 by $90 \%$, and blue light alone $\left(30 \mathrm{~J} / \mathrm{cm}^{2}\right)$ significantly suppressed the growth of Candida auris. Total inhibition effect was obtained in the $410 \mathrm{~nm}$ plus $\mathrm{H}_{2} \mathrm{O}_{2}(5.5 \mathrm{mM})$ treated 
a
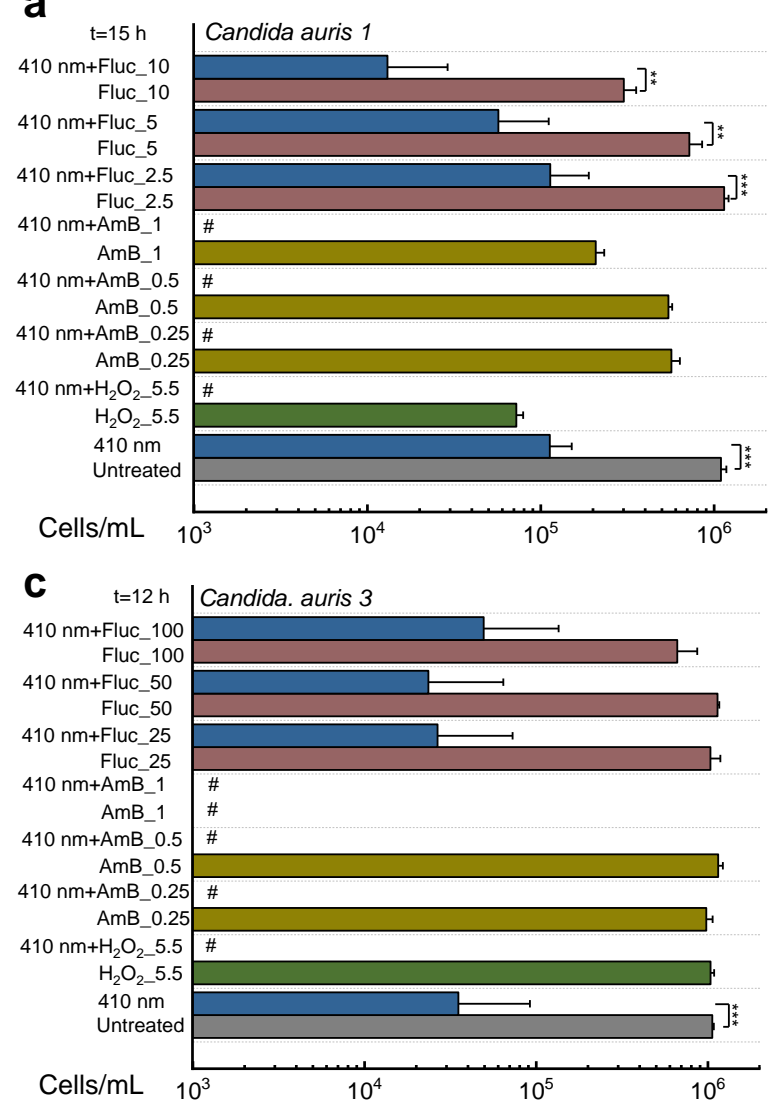

f
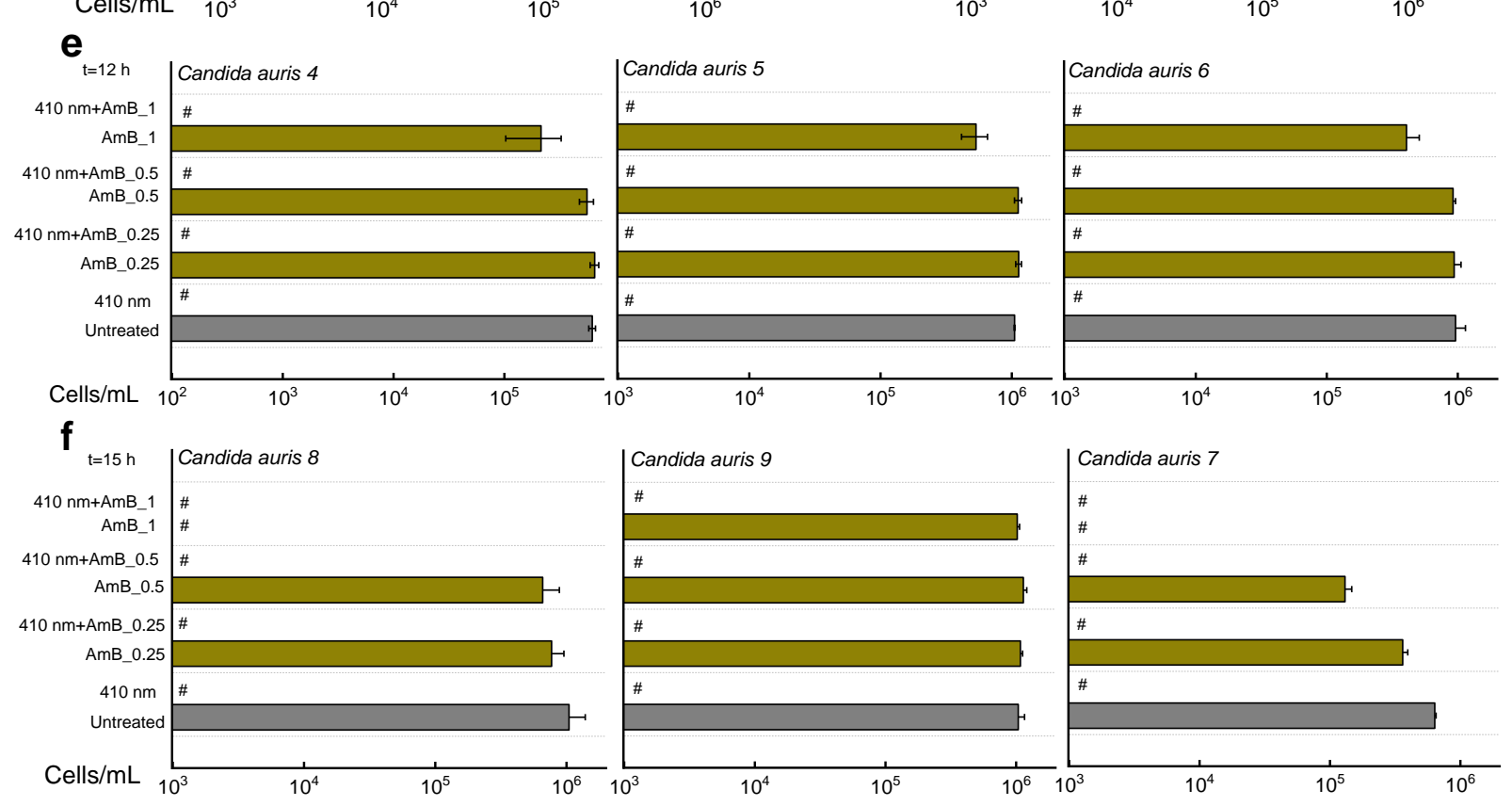

\section{b}

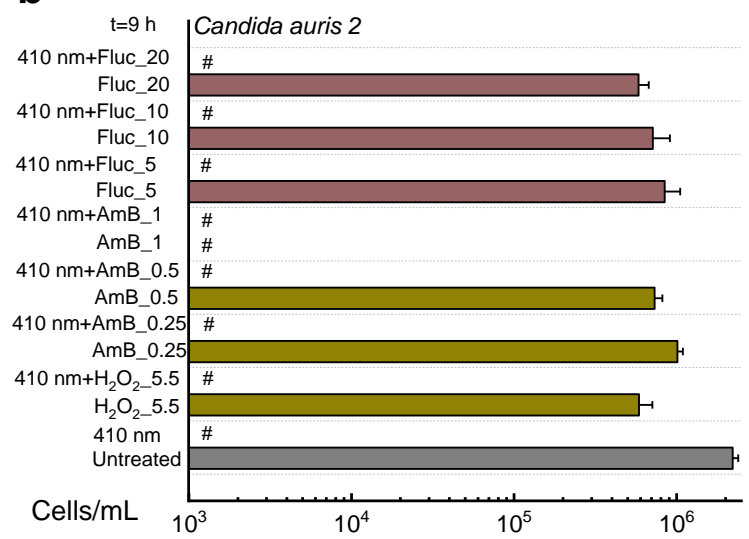

d

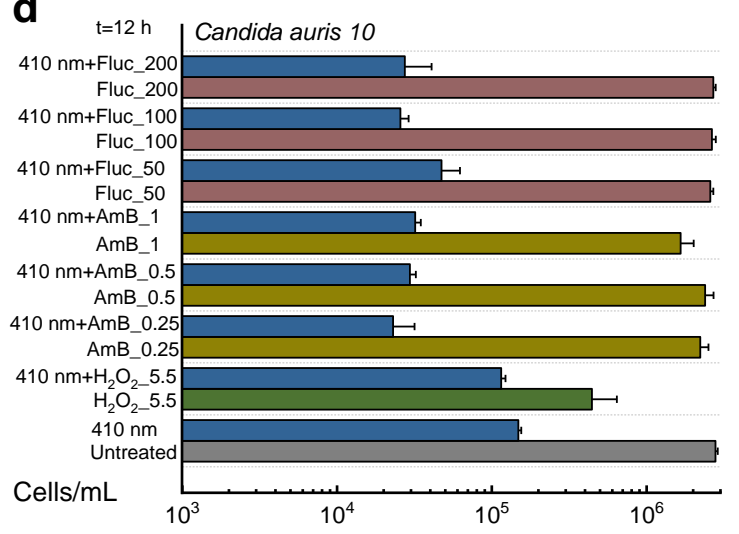

Figure 5. Derived cells/ml from ten clinical Candida auris strains based on the PrestoBlue proliferation assay and calibration curves. Data: Mean+SD. $N=3$. Abbreviations: amphotericin $B(A m B)$, fluconazole (Fluc). Significant different was determined through student unpaired $t$-test. ${ }^{*}: p<0.05,{ }^{\star *}$ : $p<0.01{ }^{* * *}: p<0.001$. Pound sign (\#) means calculated values are below the detection limit. 
group. Meanwhile, we also observed that total inhibition of proliferation happened in the $410 \mathrm{~nm}$ plus amphotericin B (AmB) treated group whereas AmB alone exerted limited efficacy. Noteworthy, photoinactivation of catalase could enhance the fungistatic effects of fluconazole by around one order of magnitude (Figure 5a). Similar phenomena were observed in the case of $C$. auris 3 (MIC of fluconazole is $128 \mu \mathrm{g} / \mathrm{ml}$ ) and C. auris 10 (MIC of fluconazole is $>256 \mu \mathrm{g} / \mathrm{ml}$ ) as well (Figure 5c-d). Strikingly, we further found that multiple $C$. auris strains are highly susceptible to $410 \mathrm{~nm}$ blue light treatment alone (Figure 5e-f) as total inhibition was observed, hinting that ample blue light-sensitive endogenous chromophores existing inside $C$. auris strains. Collectively, $C$. auris were highly sensitive towards blue light, and photoinactivation of catalase renders $C$. auris susceptible to various ROS-producing agents, even for some antifungal agents to which $C$. auris have developed resistance.

\section{Photoinactivation of catalase boosts macrophage killing of $C$. albicans}

When fungal infection occurs, pathogenic fungi will encounter our critical line of defense, the innate immune system where phagocytic macrophages can recognize, engulf, and destroy these fungal cells (45). It was reported that $C$. albicans can cause macrophage membrane rupture and lysis through hyphal germination (46). Intracellular $C$. albicans can survive and duplicate within human macrophages by harnessing array of strategies. One of such essential strategies is the expression of ROS scavenging enzymes, catalase and superoxide dismutase (45). Thus, we wondered whether photoinactivation of catalase could deprive $C$. albicans off this vital virulence factor, thus facilitating macrophage killing.

To test this hypothesis, we infected a macrophage cell line RAW 264.7 with untreated $C$. albicans and $410 \mathrm{~nm}$ blue light-treated $C$. albicans at a multiplicity of infection of 10 for 1 hour. After that, we performed a Live (SYTO 9)/Dead (PI) staining assay to visualize intracellular $C$. albicans. As shown in Figure 6a-c, after one hour of infection, C. albicans indeed exhibited hyphal morphology, and pierced through the macrophages. A small portion of $C$. albicans remained alive inside the macrophages (Figure 6a). By comparison, in the $410 \mathrm{~nm}$ blue light-treated group, we not only observed significantly less $C$. albicans in their hyphal form, but also significantly shortened hyphal length (Figure 6d-f). Quantitative analysis of the hyphal length under different treatment groups (Figure 6g) further consolidated this finding. Furthermore, there is a significant reduction of $C$. albicans viability after macrophage phagocytosed $410 \mathrm{~nm}$ pre-exposed $C$. albicans (Figure 6h). Collectively, these evidences suggest that photoinactivation of catalase attenuates the virulence of $C$. albicans, thus boosting macrophage elimination of intracellular C. albicans. Of note, blue light $(410 \mathrm{~nm})$ treatment did not pose significant toxicity to $\mathrm{CHO}$ cells (Figure 6i) under the same dosage.

It was also reported that catalase is an indispensable enzyme for $C$. albicans hyphal formation (47). Therefore, there are two plausible explanations for the photoinactivation of catalase-mediated macrophage killing. On one hand, photoinactivation of catalase might inhibit hyphal formation through catalase depletion, thus causing less macrophage rupture and lysis; one the other hand, ROS from macrophages can efficiently exert its antimicrobial effect against intracellular $C$. 
albicans without the protection of catalase. In short, photoinactivation of catalase holds the clinical potential for eliminating intracellular fungal cells.

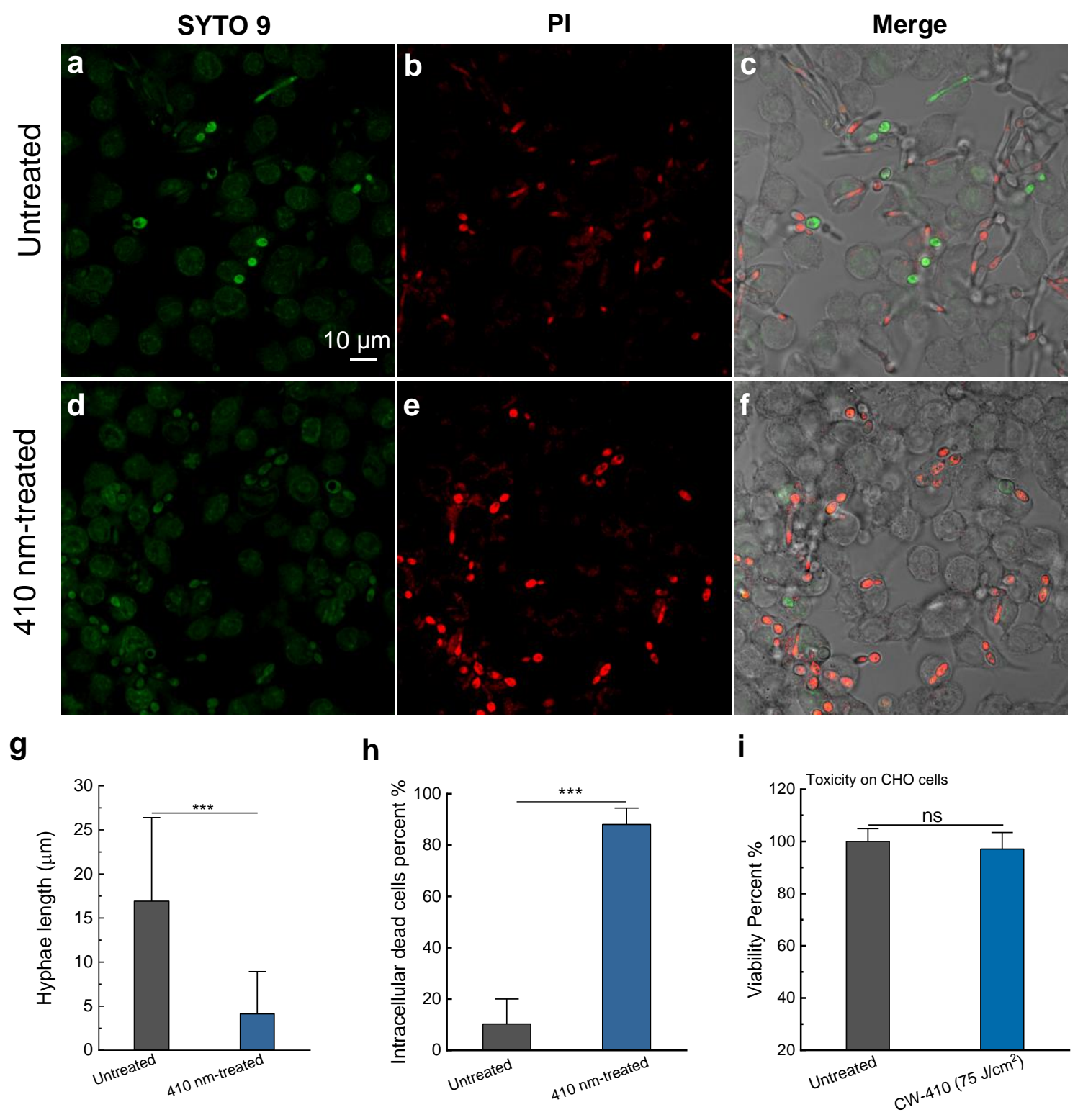

Figure 6. Elimination of intracellular C. albicans SC5314 by synergizing photoinactivation of catalase with host innate immune cells. a-f. Confocal imaging of live/dead C. albicans SC5314 after C. albicans SC5314 (a-c) infection of macrophages RAW 264.7 with or without $410 \mathrm{~nm}$ blue light pre-treatment for 1 hour at a multiplicity of infection of 10. g. Quantitative analysis of Candida hyphae length of the above two groups. h. Quantitative analysis of intracellular dead bacteria percent of the above two groups. i. CHO cell viability with or without $410 \mathrm{~nm}$ blue light treatment by MTT assay. Data: Mean+SD. N=3. Student unpaired $t$-test. ${ }^{*}: p<0.05,{ }^{* *}: p<0.01{ }^{* \star *}: p<0.001$. 


\section{Photoinactivation of catalase reduces fungal burden in a $C$. albicans-induced mouse abrasion model}

With these in vitro implications, we query the clinical utilization of photoinactivation of catalase against fungal infections. It was reported that $C$. albicans-caused infections usually start from superficial infections before developing severe systematic infections (48). Therefore, we chose a $C$. albicans infected mouse skin abrasion model (49) to evaluate our the treatment efficacy in vivo. Briefly, $10^{6} \mathrm{CFU}$ of $C$. albicans SC5314 was applied to abraded mouse skin for three hours, after that different treatment schemes $\left(120-\mathrm{J} / \mathrm{cm}^{2} 410 \mathrm{~nm}\right.$ blue light, $0.5 \% \mathrm{H}_{2} \mathrm{O}_{2}, 410 \mathrm{~nm}$ blue light plus $0.5 \% \mathrm{H}_{2} \mathrm{O}_{2}$ ) were then administered to the infected wounds (Figure 7a). Two hours after the second dose, mice were euthanized, and infected wounds homogenized and serially diluted onto C. albicans-specific BiGGY agar plates (50).

As shown in Figure 7b, C. albicans isolated from infected wounds were depicted as smooth black colonies on the BiGGY agar plates. Under the same dilution factor, $410 \mathrm{~nm}$ plus $0.5 \% \mathrm{H}_{2} \mathrm{O}_{2}$ treated group apparently showed the lowest number of $C$. albicans colonies compared to the other treatment groups. Quantitative analysis of CFU/ml from homogenized wounds further confirmed that the fungal burden in the $410 \mathrm{~nm}$ blue light plus $0.5 \% \mathrm{H}_{2} \mathrm{O}_{2}$ treated group was consistently the lowest compared to the other three groups (Figure 7c). These results suggest that photoinactivation of catalase is effective in reducing overall fungal burden in the clinical-relevant settings.

To interrogate whether the above treatment induces skin damage, we examined and further compared the normal mouse skin with or without $410 \mathrm{~nm}$ blue light $\left(120 \mathrm{~J} / \mathrm{cm}^{2}\right)$ plus $\mathrm{H}_{2} \mathrm{O}_{2}$ treatment through hematoxylin and eosin $(\mathrm{H} \& \mathrm{E})$ staining analysis. As shown in Figure 7d, epidermis, dermis, and subcutaneous tissues from the treated mouse skin appeared as intact as that of untreated one, indicating that there is no detectable toxicity from the $410 \mathrm{~nm}$ blue light (120 $\mathrm{J} / \mathrm{cm}^{2}$ ) plus $\mathrm{H}_{2} \mathrm{O}_{2}(0.5 \%)$ treatment.

Meanwhile, we also employed H\&E staining assay to visualize $C$. albicans infected mice skin from the above treatment groups. Yeast-form $C$. albicans turned into hyphal structures after seventeen hours of infection (Figure 7e), consistent with the fact that $C$. albicans usually transitions from yeast to hyphae form in disease settings (51). Of note, neutrophils or macrophages infiltration likely happened in the area adjacent to fungal infection site based on the histology analysis, further immunohistochemistry staining analysis is needed to understand which specific immune cells phagocytosed $C$. albicans. Strikingly, in the groups involved with $410 \mathrm{~nm}$ blue light treatment, hyphae-form $C$. albicans barely showed up. Only few $C$. albicans remained on the mice skin surface after $410 \mathrm{~nm}$ plus $0.5 \% \mathrm{H}_{2} \mathrm{O}_{2}$ treatment. Collectively, photoinactivation of catalase reduces the virulence of $C$. albicans, thus rendering this pathogen highly susceptible to exogenous $\mathrm{H}_{2} \mathrm{O}_{2}$ attack in a clinically relevant mice fungal infection model. 
a

Balb/c mice
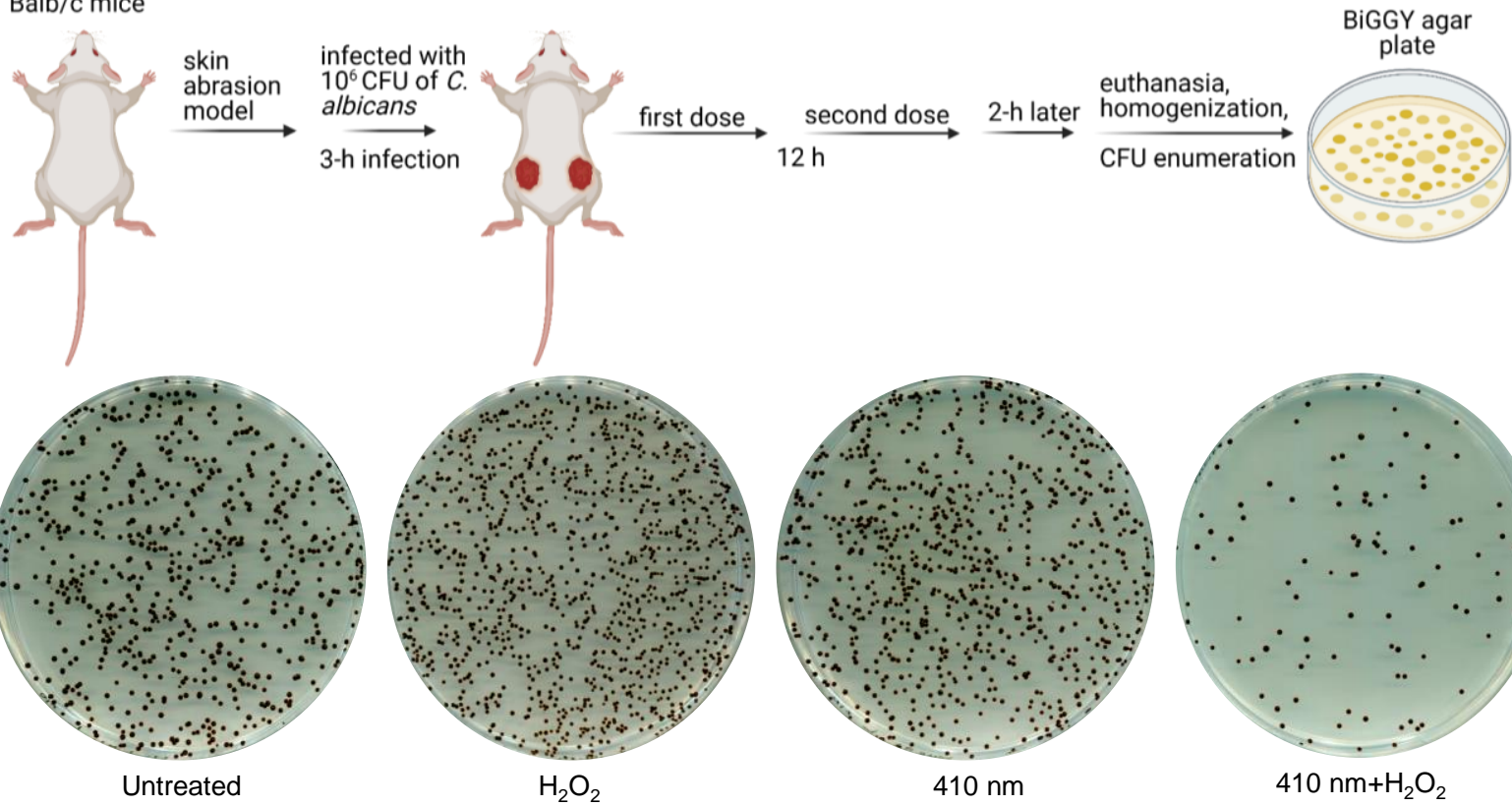

b

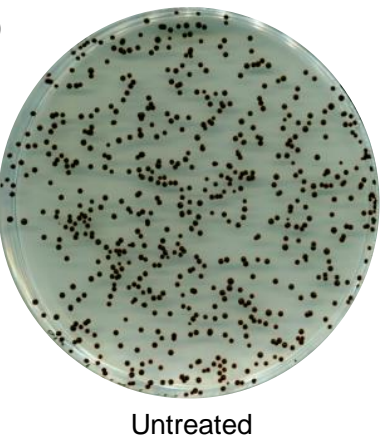

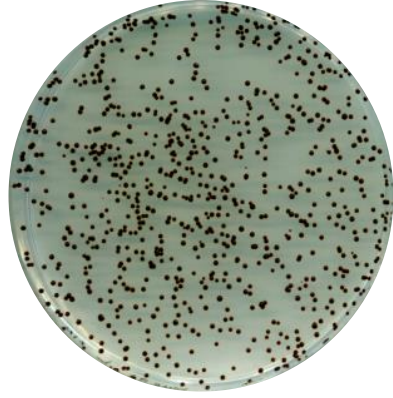

$410 \mathrm{~nm}$

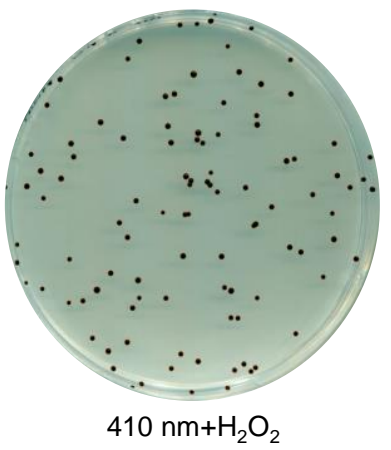

C

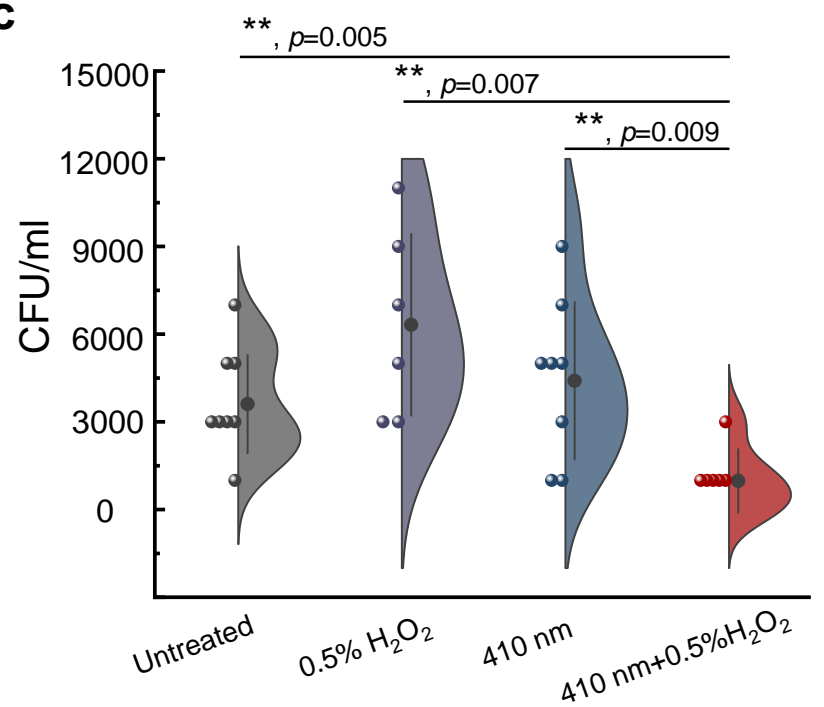

d
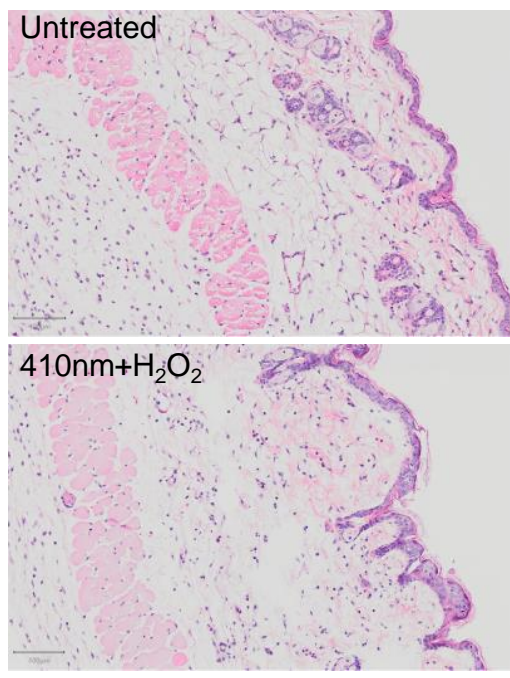

e

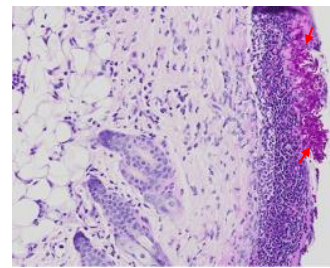

Untreated

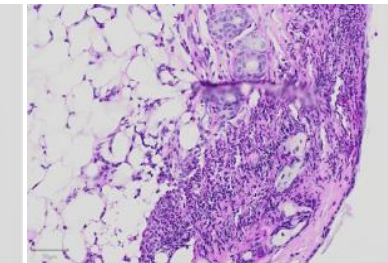

$\mathrm{H}_{2} \mathrm{O}_{2}$

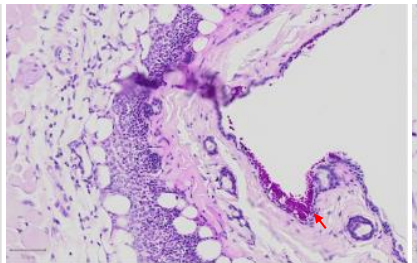

$410 \mathrm{~nm}$

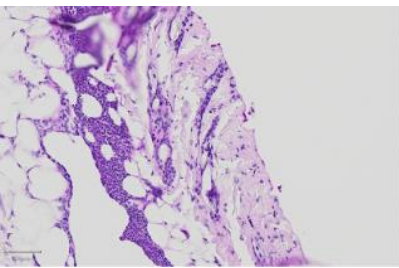

$410 \mathrm{~nm}+\mathrm{H}_{2} \mathrm{O}_{2}$

Figure 7. Photoinactivation of catalase and $\mathrm{H}_{2} \mathrm{O}_{2}$ synergistically reduces $C$. albicans burden in a mice skin abrasion model. a. Schematic illustration of development and subsequent treatment for $C$. albicansinduced mice skin abrasion. b. Spread BiGGY agar plates (Candida specific) from homogenized mice wounds after different treatment schemes. c. C. albicans $\mathrm{CFU} / \mathrm{ml}$ of the homogenized mice wounds after different treatments in (a). d. H\&E stained histology slides of the untreated mice skin and $410 \mathrm{~nm}$ blue light 
plus $\mathrm{H}_{2} \mathrm{O}_{2}$ treated mice skin. Scalar bar, $100 \mu$ m. e. $\mathrm{H} \& \mathrm{E}$ stained histology slides of $C$. albicans infected mice skins under different treatment schemes. Scalar bar, $50 \mu \mathrm{m}$. Fungal colonization area was pointed by red arrows. Data: Mean+SE from at least six replicates. Significant difference was determined through student unpaired $t$-test. ${ }^{*}: p<0.05,{ }^{* *}: p<0.01 ;{ }^{* *}: p<0.001$. Outlier was determined through whisker box plot.

\section{Consecutive blue light treatment does not induce resistance development by C. albicans}

In most of clinical settings, multiple doses of treatments are usually necessary to ensure sufficient clearance of fungal cells. To understand whether continuous $410 \mathrm{~nm}$ treatment could induce fungal resistance, we examined the performance of $C$. albicans after six-consecutive-day blue light treatment (Figure 8a). First, CFU enumeration assay was performed on C. albicans at day 0, 3, 6 under different treatment schemes. Next, we compared the catalase amount of $C$. albicans at day 0 and day 6 through the Amplex Red catalase kit.

As shown in Figure 8b, C. albicans from day 0, 3, 6 demonstrated similar sensitivity towards $\mathrm{H}_{2} \mathrm{O}_{2}$ treatment, and total eradication of $C$. albicans in the $410 \mathrm{~nm}$ blue light plus $\mathrm{H}_{2} \mathrm{O}_{2}$ treated group was achieved consistently. Quantitative analysis of CFU/ml results further consolidated the results, indicating that $C$. albicans doesn't develop resistance towards consecutive blue light treatments.

To understand whether catalase amount has any difference before and after the consecutive treatments, we compared the catalase amount inside $C$. albicans at day 0 and day 6 using the Amplex Red catalase kit. Interestingly, we didn't find significant difference between these two groups (Figure 8c), further suggesting that catalase, the molecular target of $410 \mathrm{~nm}$ blue light, is consistently expressed throughout the serial passage, and it is unlikely to develop resistance towards $410 \mathrm{~nm}$ blue light-based therapy. 
a

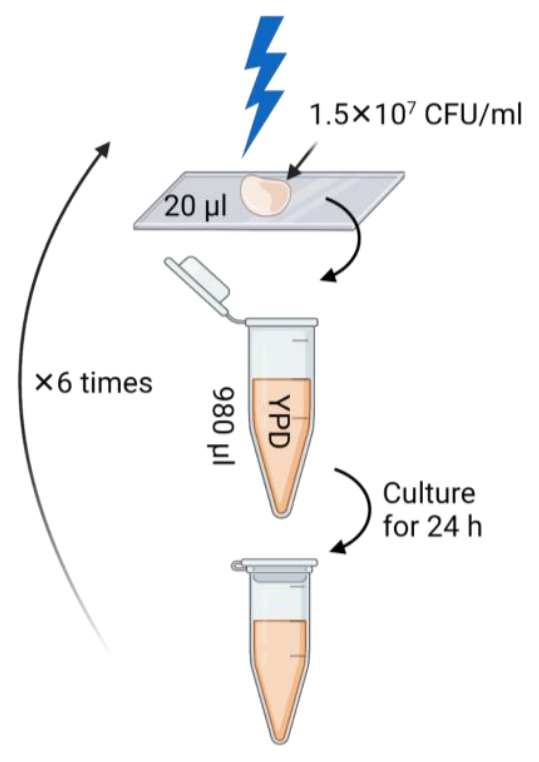

b

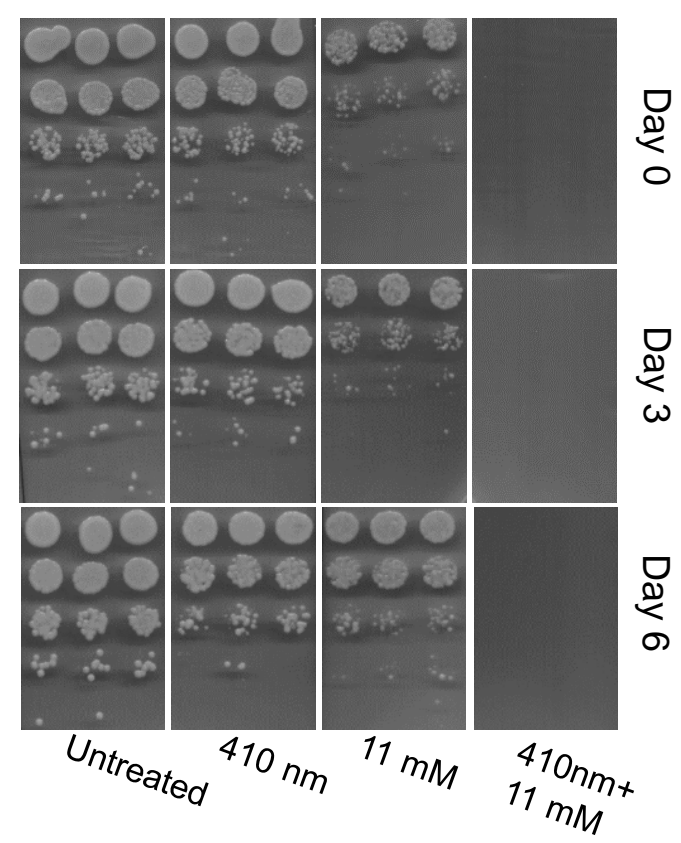

C

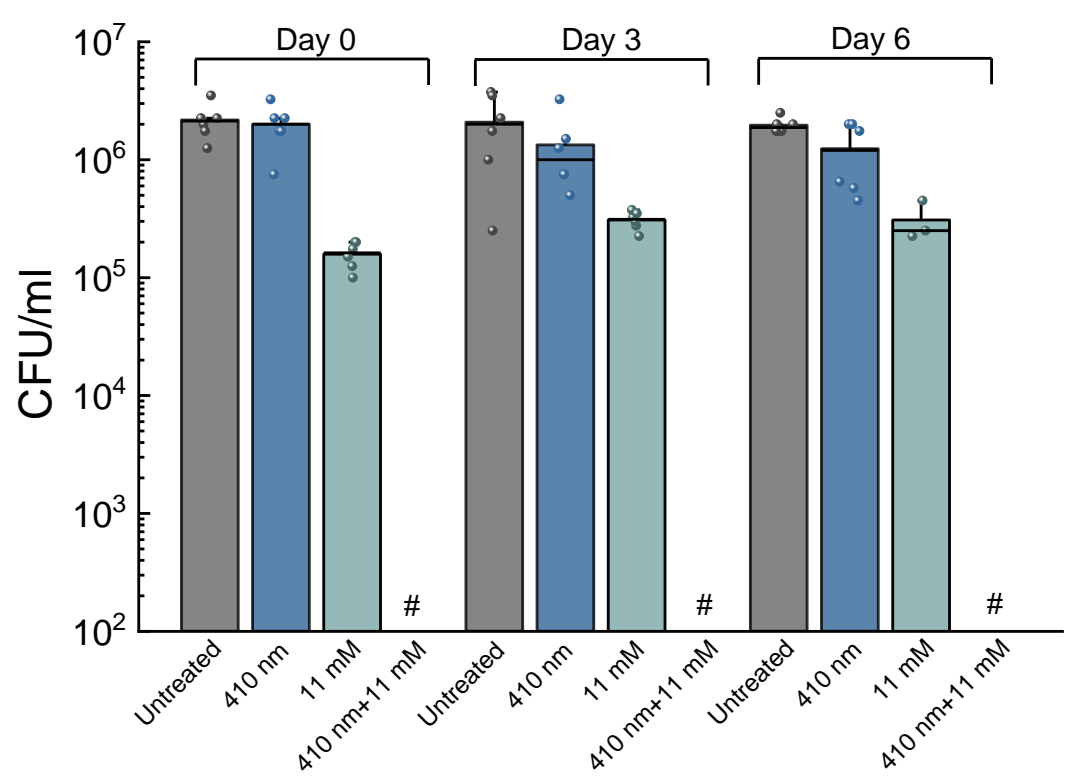

d

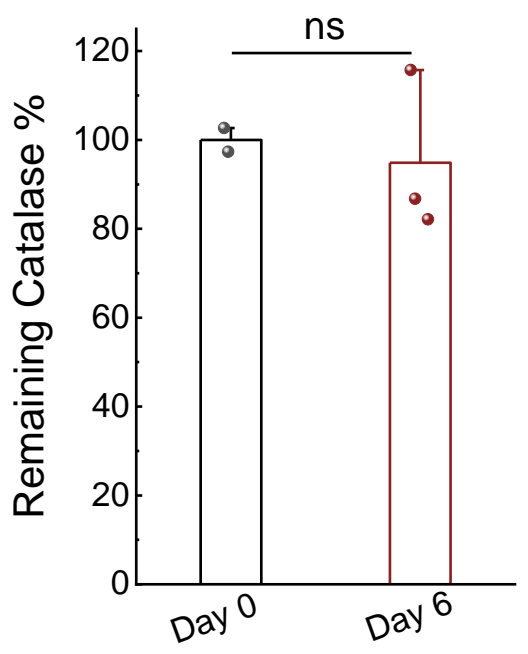

Figure 8. Candida albicans doesn't develop resistance towards consecutive blue light treatments. a. Schematic illustration of the serial blue light treatment and passage experiment. b. Spread plates of CFU of C. albicans from Day 0, 3, 6 under different treatment schemes. c. Quantitative analysis of C. albicans $\mathrm{CFU} / \mathrm{ml}$ under various treatments. Pound sign (\#) indicates the CFU results are below the detection limit. d. Catalase amount comparison from $C$. albicans before and after six-consecutive blue light exposure. Student unpaired $t$-test. ns: not significant. $410 \mathrm{~nm}: 60 \mathrm{~J} / \mathrm{cm}^{2}$. 


\section{Discussion}

Antifungal resistance has raised alarms and threats to clinicians and patients due to the limited arsenal of antifungal agents as well as current impeded antifungal development pipeline (52). For severely ill or immunocompromised patients, this situation especially worsens, and systematic candidiasis or candidemia might happen. Association of invasive aspergillosis accompanying COVID-19 patients as secondary infection have been reported (53). In addition, the global overuse of antifungals accelerates development of multidrug or pan-drug resistant fungal cells. Therefore, novel approaches to combat fungal infection are highly desired. Here, using wild-type $C$. albicans along with catalase-deficient $C$. albicans, we first identified catalase residing inside wide-ranging fungal species as a primary target of blue light. Its function in detoxifying intracellular $\mathrm{H}_{2} \mathrm{O}_{2}$ can be efficiently inactivated by blue light, especially around $410 \mathrm{~nm}$ range, which subsequently renders these fungal species highly susceptible to ROS-producing agents. In addition, the synergy between photoinactivation of catalase and $\mathrm{H}_{2} \mathrm{O}_{2}$ remains effective for various fungal species and strains, especially, the notorious clinical drug-resistant $C$. auris strains. Towards clinical applications, we found that photoinactivation of catalase boosts macrophage killing of intracellular C. albicans, and reduces C. albicans burden in a C. albicans infected mouse model of skin abrasion once combining with hydrogen peroxide. Consecutive blue light passage study indicates that neither catalase expression level nor cell susceptibility to blue light is changed for serial blue light treatment. These findings suggest the great potential of catalase-targeting blue light therapy as a new alternative to treat clinical multidrug resistant fungal infections.

Of note, blue light-mediated inactivation of $C$. albicans has been documented before (13). However, the underlying molecular target and the eradication mechanism for blue light are still unclear, which seriously hinders follow-up developments in improving its efficacy as well as its clinical translations. Based on our studies here we unveiled that blue light alone only exerts limited efficacy by inactivating catalase and ROS-producing agents are essential to be administered as adjuvants to synergize with $410 \mathrm{~nm}$ blue light for strikingly improved fungal eradication. For example, the light dose and settings we applied here are well below the ANSI standard (200 $\mathrm{mW} / \mathrm{cm}^{2}$ for $410 \mathrm{~nm}$ ), and blue light-mediated photoinactivation of catalase sensitizes $C$. albicans to non-fungicidal low-concentration $\mathrm{H}_{2} \mathrm{O}_{2}$ by around six orders of magnitude. As another example, $\mathrm{AmB}$, the golden standard for antimycotic treatment for the most severe fungal infections (54), exhibits part of its killing efficacy through ROS burst after binding with ergosterol (55). However, high-dose AmB treatment often leads to acute renal failure as a well-documented serious complication (56). Our findings suggest an alternative approach to tune down the usage of AmB by photoinactivation of catalase. Moving further, photoinactivation of catalase also revives certain antifungal agents such as fluconazole against azole-resistant clinical $C$. auris strains (Figure 5cd).

We found that multiple clinical $C$. auris strains are exceptionally sensitive to blue light when compared to the other fungal cells as total growth inhibition was obtained through PrestoBlue proliferation assay (Figure 5). However, it remains enigmatic regarding exactly why C. auris has such high sensitivity to blue light. Likely, it possesses relatively higher amount of catalase when compared to other Candida species. Nevertheless, further quantitative characterizations of catalase 
amount and related mRNA expression levels are helpful to address this query. C. auris also demonstrates a propensity to persist on skin or environmental surfaces, a unique feature to distinguish it from other Candida species, thus highly transmissible (57). Our data here demonstrates the high susceptibility of $C$. auris to blue light, and blue light alone can be developed as a novel approach for eradicating drug-resistant $C$. auris colonized on the skin or environmental surfaces.

We also found that photoinactivation of catalase boosts macrophage killing of intracellular $C$. albicans. Particularly, shortened hyphae length was observed inside the macrophages infected by the $410 \mathrm{~nm}$ pre-exposed $C$. albicans, suggesting the pivotal role of catalase plays in the transition from yeast- to hyphae-form $C$. albicans. It has been reported catalase gene disruption did not induce hyphae formation in yeast-form Saccharomyces cerevisiae (47). Therefore, further molecular-level study to further understand how $410 \mathrm{~nm}$ blue light treatment modulates such yeastto-hyphae transformation can be pursued. In addition, significant number of yeast-form C. albicans were found dead inside macrophages, suggesting an effective $410 \mathrm{~nm}$ blue light-mediated macrophage phagocytosis. Other immune cells like neutrophils also produce high level of ROS when phagocytosing fungal cells (58). Therefore, we suspect that photoinactivation of catalase could assist neutrophils to eliminate intracellular fungal cells via a similar mechanism. Taken together, photoinactivation of catalase presumably enhances immune cells to phagocytose fungal cells, thus preventing the potential disseminated candidiasis.

In an independent and parallel study, we identified catalase in a wide range of bacterial species as a primary molecular target of blue light and further demonstrated the potential utilization of photoinactivation of catalase to eradicate various pathogenic bacteria through combination with $\mathrm{H}_{2} \mathrm{O}_{2}$-producing agents (59). The current work unveiled catalase as a primary target of blue light in wide-ranging fungal species, thus broadening the scope of utilization of photoinactivation of catalase to an even wider range of pathogenic microbes. Although catalase is identified as the primary target of blue light, we believe that other molecular targets other than catalase might still exist. This is evidenced by enhanced $\mathrm{H}_{2} \mathrm{O}_{2}$ killing of catalase-deficient $C$. albicans by $410 \mathrm{~nm}$ blue light. To identify these peripheral targets in the future, endogenous pigments could be further examined as intrinsic chromophores e.g. staphyloxanthin, have shown striking sensitivity to blue light-mediated photobleaching (60-63). It has also been reported that melanin is the major pigment inside $C$. albicans playing a myriad of biological functions (64). Photoinactivation or photomodulation of melanin might account for blue light-mediated killing as well. Another direction to pursue is to improve light penetration depth, as blue light currently can only penetrate a few hundred microns (65), thus limiting its applications only to superficial surfaces. For deepseated fungal infections, pulsed blue laser with high-peak power might offer improved and deeper penetration compared to the continuous-wave light (61). Upconverting nanoparticles, capable of converting near-infrared red excitation into visible and ultraviolet emission (66), might suggest another potential way to treat deep-tissue fungal infections. In summary, our findings here suggest a fundamental molecular target and eradication mechanism of blue light elimination of multiple fungal species. These mechanistic insights create new possibilities to find highly effective treatment avenues for surface sterilization and for treatment of superficial fungal infections. 


\section{Materials and Methods}

Blue light source: Continuous-wave $(\mathrm{CW})$ blue light was delivered through a mounted $405 \mathrm{~nm}$ blue light LED (M405L4, Thorlabs) with an adjustable collimation adapter (SM2F32-A, Thorlabs) focusing the illumination region to a $\sim 1 \mathrm{~cm}^{2}$ region. A T-Cube LED driver (LEDD1B, Thorlabs) allowed for adjustable light fluencies up to $500 \mathrm{~mW} / \mathrm{cm}^{2}$.

\section{Fungal strains and chemicals}

Fungal strains: Candida albicans SC5314 (wild type, American Type Culture Collection (ATCC)). Catalase-deficient Candida albicans 2089 ( $\triangle$ cat 1$)$ are from Dr. Alistair Brown lab at University of Exeter. All the other strains are from Dr. Michael K. Mansour's lab (clinical strains from Massachusetts General Hospital (MGH), Boston, MA) including all the Candida auris strains: Candida auris 1_MGH, Candida auris 1 (AR-0381), Candida auris 2 (AR-0382), Candida auris 3 (AR-0383), Candida auris 4 (AR-0384), Candida auris 5 (AR-0385), Candida auris 6 (AR-0386), Candida auris 7 (AR-0387), Candida auris 8 (AR-0388), Candida auris 9 (AR-0389), Candida auris 10 (AR-0390). Candida albicans C15, Candida albicans C16, Candida albicans C17. C. glabrata (ATCC 2001), Candida glabrata C1, Candida glabrata C2, C. tropicalis (H3222861), C. parapsilosis (F825987), C. lusitaniae (S1591976), C. lusitaniae (AR-0398), C. haemulonii (AR-0393), C. haemulonii (AR-0395), Candida duobushaemulonii (AR-0394), $C$. krusei (AR-0397). All cell lines used in this study, including the RAW 264.7 murine macrophages, Chinese hamster ovary $(\mathrm{CHO})$ cells were purchased directly from the ATCC.

Chemicals: Amphotericin B (A9528-100MG, Sigma Aldrich). Hydrogen peroxide (CVS Health). DMSO (D8418-500ML, Sigma Aldrich). YPD broth (Y1375, Sigma Aldrich). YPD Agar (Y1500, Sigma Aldrich). Phosphate buffered saline (BP399500, Fisher Scientific). 10\% formalin (HT501128-4L, Sigma Aldrich). Sodium chloride solution (S8776, Sigma Aldrich). Miconazole nitrate salt (M3512, Sigma Aldrich). Fluconazole (86386, Acros Organics). PrestoBlue reagent (A13261, ThermoFisher). 3-Amino-1,2,4-triazole (3-AT, A8056, Sigma Aldrich). Bovine liver catalase (C1345, Sigma Aldrich). LIVE/DEAD cell viability kit (L7007, ThermoFisher). BiGGY agar (73608, Sigma Aldrich). Amplex Red Catalase Assay (A22180, Thermo Fisher Scientific). DMEM (2186822, Gibco). HI FBS (2273356P, Gibco). HEPES (54457, Sigma Aldrich).

Fungal culture: $C$. albicans was routinely streaked at $30^{\circ} \mathrm{C}$ onto the yeast peptone dextrose (YPD) agar and sub-cultured in YPD broth overnight to stationary phase. Stationary-phase Candida strains were cultured into mid-log phase prior use by 1:20 dilution by YPD broth. Fungal cell density was adjusted based on the optical density at $660 \mathrm{~nm}\left(\mathrm{OD}_{600}\right)$. The suspension was centrifuged, washed with phosphate-buffered saline (PBS), and re-suspended in PBS at the cell density of $10^{6} \sim 10^{7} \mathrm{CFU} / \mathrm{ml}$ before proceeding all the treatments.

Quantitation of remaining catalase percentage: Measurement of remaining catalase percentage was primarily quantified through the use of an Amplex Red Catalase Assay (A22180, Thermo Fisher Scientific) according to its standard protocol. Briefly, solutions containing catalase (either bovine liver catalase or catalase-positive fungal culture with $1 \times 10^{6}$ cell density) were treated with 
blue light, after which $25 \mu \mathrm{l}$ of the light treated solution was incubated with $40 \mu \mathrm{M}$ of $\mathrm{H}_{2} \mathrm{O}_{2}$ for two hours at $30^{\circ} \mathrm{C}$ in dark inside a 96-well plate. Following $\mathrm{H}_{2} \mathrm{O}_{2}$ treatment, $50 \mu \mathrm{l}$ of a reaction stock containing $100 \mu \mathrm{M}$ of Amplex Red and $0.4 \mathrm{U} / \mathrm{mL}$ of horseradish peroxidase were added to each well and then incubated for 30 minutes at $37^{\circ} \mathrm{C}$. Following incubation, the fluorescence of each well was measured at an excitation wavelength of $545 \mathrm{~nm}$ and an emission wavelength of $590 \mathrm{~nm}$. A negative control containing the $1 \times$ reaction buffer $(0.1 \mathrm{M}$ Tris- $\mathrm{HCl})$ and an untreated positive control (normal fungal cells without blue light exposure) also treated with the assay in order to determine the remaining catalase percentage. Remaining catalase percentage was calculated through the following equation:

$$
\text { Remaining catalase } \%=\frac{I_{\text {buffer }}-I_{\text {treated }}}{I_{\text {buffer }}-I_{\text {untreated }}} \times 100 \%
$$

\section{Imaging of intracellular $\mathrm{H}_{2} \mathrm{O}_{2}$}

C. albicans with and without $410 \mathrm{~nm}$ exposure $\left(30 \mathrm{~J} / \mathrm{cm}^{2}\right)$ were incubated with $\mathrm{H}_{2} \mathrm{O}_{2}(22 \mathrm{mM}, 30$ min incubation). After that, $C$. albicans were stained with an intracellular $\mathrm{H}_{2} \mathrm{O}_{2}$ kit (MAK164$1 \mathrm{KT}$ ) for $30 \mathrm{~min}$. Fungal cells were then washed and sandwiched between a cover slide and polylysine-coated cover glass. Confocal laser scanning microscopy was then applied to image intracellular $\mathrm{H}_{2} \mathrm{O}_{2}$.

Bubbling test, CFU enumeration assay, short-term incubation assay, Checkerboard broth dilution assay and time killing assay to validate the mechanism of catalase inactivation in catalase inhibited or knocked-out Candida strains: Candida albicans SC5314 and Candida albicans $\Delta$ catl were cultured overnight in YPD broth at $30^{\circ} \mathrm{C}$ using an orbital shaking incubator (250 rpm).

For the bubbling test, after overnight culturing, both the wild type C. albicans SC5314 and Candida albicans $\Delta$ catl were washed by PBS and adjust the cell density to $10^{7} / \mathrm{ml}$, placed $1 \mathrm{ml}$ of fungal solution into cuvettes with $80 \mu \mathrm{l}$ of $3 \% \mathrm{H}_{2} \mathrm{O}_{2}$, and then incubated at room temperature for $30 \mathrm{~min}$ before observation.

For the CFU enumeration, the next day after overnight culture, fungal cultures were resuspended 1:20 in YPD for another 5 6 hours and cultured into mid-log phase. Then fungal cells were washed, suspended in PBS and adjusted at the optical density by $660 \mathrm{~nm}\left(\mathrm{OD}_{660}\right)$ to $\sim 10^{8} \mathrm{CFU} / \mathrm{ml}$, after which a $10 \mu \mathrm{l}$ of aliquot was placed on a glass cover slide and then treated with CW-410 nm $\left(100 \mathrm{~mW} / \mathrm{cm}^{2} 5 \mathrm{~min}\right)$. Following light treatment, the droplet was collected to a tube containing PBS or PBS supplemented with $\mathrm{H}_{2} \mathrm{O}_{2} . \mathrm{H}_{2} \mathrm{O}_{2}$ ranged from $0.69 \mathrm{mM}$ to $22 \mathrm{mM}$ according to the sensitivity of fungal strains. Samples were then incubated for 30 minutes at $30^{\circ} \mathrm{C}$ in the orbital shaking incubator, after which the treated and untreated fungal samples were 10-fold serially diluted inside a 96-well plate, plated on YPD agar plates in a $30^{\circ} \mathrm{C}$ static incubator for $24 \sim 36$ hours prior to enumeration. All the CFU results were read at the same time for each patch of experiment. 
Short-term incubation assay: In order to determine the impact of short term $\mathrm{H}_{2} \mathrm{O}_{2}$ exposure on C. auris following light induced catalase inactivation, C. auris 2 was incubated overnight in YPD broth. The next day $200 \mu \mathrm{l}$ of $C$. auris was centrifuged and resuspended in $1 \mathrm{ml}$ of $1 \times \mathrm{PBS}$. A 10 $\mu \mathrm{l}$ aliquot of $C$. auris suspension was placed on a glass coverslip and exposed to $30 \mathrm{~J} / \mathrm{cm}^{2}$ of CW$410\left(200 \mathrm{~mW} / \mathrm{cm}^{2}, 2.5 \mathrm{~min}\right)$. Following exposure, the aliquot was transferred to $990 \mu \mathrm{l}$ of PBS and vortexed. This process was repeated for the non-light treated $C$. auris as well. After the creation of the light treated and untreated $C$. auris stock, $300 \mu$ l of stock was separated and treated with $0.1 \% \mathrm{H}_{2} \mathrm{O}_{2}$ for $1 \mathrm{~min}$, after which the $\mathrm{H}_{2} \mathrm{O}_{2}$ treated suspension was serially diluted and plated to determine changes in overall $\mathrm{CFU}$ following exposure.

For the catalase inhibition assay, 3-Amino-1,2,4-triazole (3-AT) was applied to both untreated and $\mathrm{H}_{2} \mathrm{O}_{2}$ containing groups at the concentration $50 \mathrm{mM}$ ), and cultured with $C$. albicans SC5314 for 4 hours in a $30^{\circ} \mathrm{C}$ shaking incubator. After treatment, the treated suspensions were spun down and resuspended in fresh PBS, after which a $10 \mu \mathrm{l}$ aliquot of untreated $C$. albicans was treated with $30 \mathrm{~J} / \mathrm{cm}^{2}$ of CW-410 $\left(100 \mathrm{~mW} / \mathrm{cm}^{2}, 5 \mathrm{~min}\right)$ and then diluted in $990 \mu \mathrm{l}$ of PBS. Alongside the light treated dilution, an untreated dilution and 3-AT treated dilution were also produced. These dilutions were treated with $44 \mathrm{mM}$ of $\mathrm{H}_{2} \mathrm{O}_{2}$ for $2 \mathrm{~h}$ at $30^{\circ} \mathrm{C}$, after which the samples were serially diluted and plated to quantify the overall $\mathrm{CFU}$ enumeration.

For the time killing assay, Candida albicans SC5314 and Candida albicans $\Delta$ cat1 were cultured into mid-exponential phase in YPD broth at $30^{\circ} \mathrm{C}$ using an orbital shaking incubator $(250 \mathrm{rpm})$. Then the fungal culture was washed and adjusted based on $\mathrm{OD}_{660}$ to $\sim 10^{8} \mathrm{CFU} / \mathrm{ml}$, after which a $10 \mu \mathrm{L}$ aliquot was placed on a glass cover slide and then treated with CW-410 nm $\left(100 \mathrm{~mW} / \mathrm{cm}^{2}\right.$, 5 min). Following light treatment, the droplet was collected to a tube containing PBS or PBS containing $11 \mathrm{mM} \mathrm{H}_{2} \mathrm{O}_{2}$. Samples were shaken and incubated at $30^{\circ} \mathrm{C}$ for up to 4 hours. During the incubation, $60 \mu \mathrm{L}$ aliquots were removed from each sample tube for serial dilution and CFU plating at the $0 \mathrm{~min}, 20 \mathrm{~min}, 40 \mathrm{~min}, 1 \mathrm{~h}, 1.5 \mathrm{~h}, 2 \mathrm{~h}$ and $4 \mathrm{~h}$ time points.

For the checkerboard assay, Candida albicans SC5314 and Candida albicans $\Delta$ cat1 were cultured into mid-exponential phase in YPD broth. Then fungal culture was washed and adjusted based on $\mathrm{OD}_{660}$ to $\sim 10^{7} \mathrm{CFU} / \mathrm{ml}$ as stock solution and stored on ice. An aliquot of $5 \mu \mathrm{l}$ fungal stock solution was exposed with $410 \mathrm{~nm}$ light at a power of $500 \mathrm{~mW} / \mathrm{cm}^{2}$ for $0 \mathrm{~min}, 2.5 \mathrm{~min}, 5 \mathrm{~min}, 10$ min, $20 \mathrm{~min}, 40 \mathrm{~min}$, then collected with $995 \mu \mathrm{l}$ of YPD broth to a final cell density of $\sim 5 \times 10^{4} / \mathrm{ml}$ and plated into a 96-well plate in row. Then a two-fold serial dilution was performed from $22 \mathrm{mM}$ of $\mathrm{H}_{2} \mathrm{O}_{2}$ to the following concentrations: $11 \mathrm{mM}, 5.5 \mathrm{mM}, 2.8 \mathrm{mM}, 1.4 \mathrm{mM}, 0.7 \mathrm{mM}, 0.4 \mathrm{mM}, 0.2$ $\mathrm{mM}, 0 \mathrm{mM}$. Time-course $\mathrm{OD}_{660}$ was recorded by the plate reader over 18 hours. The combinational behavior between blue light and $\mathrm{H}_{2} \mathrm{O}_{2}$ from both wild type $C$. albicans and catalase mutant was then calculated based on the following equation:

$$
F I C=\frac{[A]}{[M I C]_{A}}+\frac{[B]}{[M I C]_{B}}
$$

Where the FIC stands for fractional inhibitory concentration, indicating the synergy level of two treatments. (FIC < 0.5: Synergy; FIC > 4: Antagonism; $0.5<$ FIC $<4$ : Additive or indifference) 


\section{Time-course PrestoBlue proliferation assay of log phased and stationary phased Candida species}

Clinical fungal strains from MGH Dr. Michael K. Mansour's lab were used in this part to test the broad band of our phototherapy, and a high throughput resazurin-based PrestoBlue assay was applied. Both stationary- and exponential phase fungal cells are included as well. Stationary phase Candida species were prepared by overnight culturing at $30^{\circ} \mathrm{C}$ using an orbital shaking incubator (250 rpm); and the log phase fungal cells were prepared the next day by 1:20 in YPD for another 5 6 hours and cultured into mid-log phase, some of the $C$. auris strains took longer time to grow. All cells were collected and washed twice, then the cell density was adjusted to $\sim 10 \% / \mathrm{ml}$ prior to blue light exposure. A $10 \mu \mathrm{l}$ of aliquot was placed onto a glass cover slide and then treated with $\mathrm{CW}-410 \mathrm{~nm}\left(100 \mathrm{~mW} / \mathrm{cm}^{2}, 5 \mathrm{~min}\right)$ or not. Following light treatment, the droplet was collected to a tube containing $990 \mu \mathrm{l}$ of PBS to reach the fungal cell density of $\sim 10^{6} / \mathrm{ml} \mathrm{H}_{2} \mathrm{O}_{2}$ ranging from 11 $\mathrm{mM}$ to $44 \mathrm{mM}$ was added to the wells in the first row and a two-fold dilution was conducted, the initial concentrations were chosen according to the sensitivity of fungi strains. Samples were then incubated for 4 hours at $30^{\circ} \mathrm{C}$, after which an aliquot of $90 \mu \mathrm{l}$ of YPD and $10 \mu \mathrm{l}$ of PrestoBlue reagent were added to each well, followed by a time-course recording of the fluorescence signal (Excitation: $590 \mathrm{~nm}$ ) for $24 \mathrm{~h}$. Calibration growth curves with different initial cell density was also conducted in order to calculate the original CFU of all the Candida strains.

\section{Intracellular fungi assay}

To assess the fungal intracellular killing with murine macrophage RAW 264.7 cell line, C. albicans SC5314 was collected from an exponentially growing culture and washed with PBS. Macrophages were cultured in Dulbecco's Modified Eagle medium (DMEM) alongside 10\% fetal bovine serum (FBS) until 90\% confluence was achieved. Macrophages were pre-washed with serum-free DMEM media immediately before infection, and infected by $C$. albicans SC5314 (MOI=10) with and without $410 \mathrm{~nm}$ treatment $\left(35 \mathrm{~mW} / \mathrm{cm}^{2}, 8 \mathrm{~min}\right)$. Then co-culture was incubated at $37^{\circ} \mathrm{C}$ in a humidified incubator to allow for the phagocytosis of fungal cells. After two hours, the infection co-culture was removed and replaced with normal growth media (DMEM supplemented with $10 \%$ FBS, $10 \mathrm{mM}$ HEPES). A Live (SYTO 9)/Dead (PI) staining assay was to utilize to visualize the live and dead fungal cells inside macrophages, respectively. Briefly, after fixation with $10 \%$ formalin following infections, samples were permeabilized with $0.1 \%$ Triton-X for 3 min at room temperature. After that, a Live/Dead fluorescence kit (Thermo Fisher Scientific, L7007) was utilized to stain the intracellular fungal cells and confocal laser scanning microscope (FV3000, Olympus) was employed to visualize the stained samples.

Mammalian cell toxicity assay: To evaluate the potential toxicity of $410 \mathrm{~nm}$ exposure and short term, high concentration $\mathrm{H}_{2} \mathrm{O}_{2}$ exposure against mammalian cells, an MTT assay was performed using Chinese Hamster Ovary (CHO) cell line. CHO cells were cultured in Dulbecco's Modified Eagle medium (DMEM) and 10\% fetal bovine serum (FBS) until a high confluence was obtained. $\mathrm{CHO}$ cells were then removed via trypsin, quantified, and diluted in serum-free DMEM media to a cell concentration of $1 \times 10^{6}$ cells $/ \mathrm{ml}$. In a treated 96 -well plate, $100 \mu \mathrm{l}$ of cell suspension was added to each well, providing $1 \times 10^{5}$ cells per well. The cells were allowed to adhere overnight at $37^{\circ} \mathrm{C}$ with $5 \% \mathrm{CO}_{2}$. 
The next day, the media was removed from each well and the cells were washed twice with PBS to minimize potential reactions between the phenol red present in DMEM and the blue light treatment. Washing conditions performed on one set of cells were also performed on all other sets to maintain consistency. Following washing, all wells were filled with PBS and the light treated wells were then exposed to $30 \mathrm{~J} / \mathrm{cm}^{2}$ of CW-410 $\left(200 \mathrm{~mW} / \mathrm{cm}^{2}, 2.5 \mathrm{~min}\right)$ After light treatment, PBS was removed and replaced with DMEM. For the $\mathrm{H}_{2} \mathrm{O}_{2}$ treated groups, $0.1 \% \mathrm{H}_{2} \mathrm{O}_{2}$ in DMEM was added to the $\mathrm{H}_{2} \mathrm{O}_{2}$ treatment groups for $1 \mathrm{~min}$, after which the media was immediately removed and the wells were washed three times with $\mathrm{PBS}$ to minimize potential remaining $\mathrm{H}_{2} \mathrm{O}_{2}$ remaining. Three-fold washing was also applied to the other treatment groups. After washing, the wells were filled with $100 \mu \mathrm{l}$ of fresh DMEM and the cells were allowed to recover from treatment over the course of 24 hours at $37^{\circ} \mathrm{C}$ with $5 \% \mathrm{CO}_{2}$. Once 24 hours have passed, an MTT viability assay was performed, where the DMEM was replaced with fresh DMEM alongside $0.5 \mathrm{mg} / \mathrm{ml}$ of MTT (Life Technologies, M6494) and incubated at $37^{\circ} \mathrm{C}$ for $4 \mathrm{~h}$. Once upon completion, the DMEM/MTT solution was removed and $100 \mu \mathrm{l}$ of filtered DMSO was mixed in and provided with 45 min to dissolve the crystalized formazan, after which absorbance measurements were quantified via plate reader at $590 \mathrm{~nm}$. The assay was performed in replicates of $\mathrm{N}=4$.

In vivo murine infection model and histology: Animal studies were approved by the Institutional Animal Care and Use Committee (IACUC) at Boston University and were performed in the Animal Science Center (ASC) in the Charles River Campus (CRC). The abrasion fungal infection model was used. Twenty BALB/c mice (Jackson Laboratories, 000651) were shaved on the dorsal side and placed under anesthesia, then a sterile \#15 sterile scalpel was used to generate two $\sim 1 \times 1$ $\mathrm{cm}^{2}$ abrasion wounds on both left and right by carefully scraping the epidermis of the skin until reddish dots appeared without drawing blood out of skin barrier. Once wounds were generated, a $10 \mu \mathrm{l}$ aliquot containing $2 \times 10^{8} / \mathrm{ml}$ of exponential phase $C$. albicans SC5314 in PBS was placed onto the abrasion wound, and spread evenly across the wound with a pipette tip and air dried. Then 20 mice were divided into four treatment groups, each consisting of 5 mice ( $\mathrm{N}=10$ wounds in total): Untreated, $410 \mathrm{~nm}$ treated, $\mathrm{H}_{2} \mathrm{O}_{2}$ treated, and $410 \mathrm{~nm}$ plus $\mathrm{H}_{2} \mathrm{O}_{2}$ treated. Light treatment was applied by positioning mice under $200 \mathrm{~mW} / \mathrm{cm}^{2}$ (ANSI standard for human skin) $410 \mathrm{~nm}$ LEDs for $10 \mathrm{~min}$ (total $120 \mathrm{~J} / \mathrm{cm}^{2}, 5$-min exposure and 5-min break and then 5-min exposure again in order to prevent potential photodamage). For $\mathrm{H}_{2} \mathrm{O}_{2}$ treatment, $10 \mu \mathrm{l}$ of $0.5 \% \mathrm{H}_{2} \mathrm{O}_{2}$ was evenly distributed on the infected wounds and air dried. Combinational treatment consisted of the application of previously described blue light treatment $\left(120 \mathrm{~J} / \mathrm{cm}^{2}\right)$ followed immediately by the administration of $\mathrm{H}_{2} \mathrm{O}_{2}$. Treatments were applied to mice twice, the first treatment being applied 4 hours following infection, and the second treatment being applied 20 hours after the infection. 2 hours after the second treatment, mice were euthanized and wound tissue was harvested, either homogenized and serially diluted for CFU studies or fixation by $10 \%$ neutral buffered formalin for histology studies. CFU enumeration was performed on C. albicans-specific BiGGY agar plates (73608, Sigma Aldrich).

The potential phototoxicity of the treatment on the skin was evaluated by hematoxylin and eosin (H\&E) histology assay. Same assay was applied as well to examine the location of fungal cells in the infection site. The untreated mouse skin received the same two treatments as the wound 
site, and during tissue harvesting, this region was excised and preserved in $10 \%$ buffered formalin. Formalin fixed samples for both untreated and treated normal skin tissues $(\mathrm{N}=6)$ and fungi infected skin tissues were submitted to the Boston Medical Center (BMC) for histology processing, periodic acid-Schiff (PAS) staining for fungal infected skin and hematoxylin and eosin (H\&E) staining for normal skin. Histology slides were then visualized by Olympics VS120 slide scanner provided by Micro/Nano Imaging (MNI) lab in Biomedical Engineering Core Facilities under in Boston University, and then the images were visualized and analyzed by a pathologist Dr. Ivy Rosales at MGH.

\section{Statistical analysis}

Statistical analysis was conducted through student unpaired $t$-test and One-way ANOVA. ${ }^{* \star *}$ means significantly different with the $p$-value $<0.001{ }^{* *}$ means significantly different with the $p$ value $<0.01$. ${ }^{*}$ means significantly different with the $p$-value $<0.05$. ns means no significance.

\section{Acknowledgments:}

This work was partly supported by RO1AI141439 to J.-X.C, and RO1AI132638 to M.K.M. We kindly acknowledge Dr. Alistair Brown lab at University of Exeter for providing the catalasedeficient $C$. albcians strains. Research reported in this publication was also supported by the Boston University Micro and Nano Imaging Facility and the office of the director, National Institute of Health, National Institute of Health under Award Number S10OD024993.

\section{Author contributions:}

P.-T.D. and J.-X.C. conceived the synergistic therapeutic treatment between photoinactivation of catalase and $\mathrm{H}_{2} \mathrm{O}_{2}$ or certain antifungals. M.K.M. provided all the clinical fungal strains including the Candida auris strains and experimental discussions. P.-T.D. and J.H. discovered that catalase from catalase-positive fungi could be ubiquitously inactivated by $410 \mathrm{~nm}$. P.-T.D. characterized catalase photoinactivation and intracellular fungi assay. P.-T.D. and Y.W.Z. conducted the mechanism study by the catalase-deficient $C$. albicans strains. P.-T.D. and S.J. conducted the 3AT study. Z.D., N.A., P.-T.D., and Y.Z. prepared the clinical fungal samples together. P.-T.D., Y.W.Z. and J.H. conducted all the in vitro PrestoBlue assay. P.-T.D., Y.W.Z., and S.J. did data analysis of Prestoblue assay. Y.W.Z., P.-T.D. and S.J. conducted the in vivo mice abrasion experiments and histology assay. Y.W.Z. conducted the histology slides scanning and preliminary analysis. P.-T.D. and J.-X.C. co-wrote the manuscript. G.L. provided constructive suggestions over the project and manuscript. All authors read and commented on the manuscript.

\section{Competing interests:}

The authors declare that they have no competing interests. 


\section{References}

1. Ascioglu, S., Rex, J.H., de Pauw, B., Bennett, J.E., Bille, J., Crokaert, F., Denning, D.W., Donnelly, J.P., Edwards, J.E., Erjavec, Z., et al. 2002. Defining Opportunistic Invasive Fungal Infections in Immunocompromised Patients with Cancer and Hematopoietic Stem Cell Transplants: An International Consensus. Clinical Infectious Diseases 34:7-14.

2. Pappas, P.G., Rex, J.H., Sobel, J.D., Filler, S.G., Dismukes, W.E., Walsh, T.J., and Edwards, J.E. 2004. Guidelines for Treatment of Candidiasis. Clinical Infectious Diseases 38:161-189.

3. Nobile, C.J., and Johnson, A.D. 2015. Candida albicans Biofilms and Human Disease. Annual review of microbiology 69:71-92.

4. Arendrup, M.C., and Patterson, T.F. 2017. Multidrug-Resistant Candida: Epidemiology, Molecular Mechanisms, and Treatment. The Journal of Infectious Diseases 216:S445-S451.

5. $\quad$ Eldesouky, H.E., Salama, E.A., Lanman, N.A., Hazbun, T.R., and Seleem, M.N. 2020. Potent Synergistic Interactions between Lopinavir and Azole Antifungal Drugs against Emerging Multidrug-Resistant Candida auris. Antimicrobial agents and chemotherapy 65.

6. Caceres, D.H., Forsberg, K., Welsh, R.M., Sexton, D.J., Lockhart, S.R., Jackson, B.R., and Chiller, T. 2019. Candida auris: A Review of Recommendations for Detection and Control in Healthcare Settings. Journal of fungi (Basel, Switzerland) 5:111.

7. Ademe, M., and Girma, F. 2020. Candida auris: From Multidrug Resistance to Pan-Resistant Strains. Infection and drug resistance 13:1287-1294.

8. Villanueva-Lozano, H., Treviño-Rangel, R.d.J., González, G.M., Ramírez-Elizondo, M.T., LaraMedrano, R., Aleman-Bocanegra, M.C., Guajardo-Lara, C.E., Gaona-Chávez, N., Castilleja-Leal, F., Torre-Amione, G., et al. 2021. Outbreak of Candida auris infection in a COVID-19 hospital in Mexico. Clinical microbiology and infection : the official publication of the European Society of Clinical Microbiology and Infectious Diseases 27:813-816.

9. Song, G., Liang, G., and Liu, W. 2020. Fungal Co-infections Associated with Global COVID-19 Pandemic: A Clinical and Diagnostic Perspective from China. Mycopathologia 185:599-606.

10. Lu, M., Wang, S., Wang, T., Hu, S., Bhayana, B., Ishii, M., Kong, Y., Cai, Y., Dai, T., Cui, W., et al. 2021. Bacteria-specific phototoxic reactions triggered by blue light and phytochemical carvacrol. Science Translational Medicine 13:eaba3571.

11. Yin, R., Dai, T., Avci, P., Jorge, A.E.S., de Melo, W.C.M.A., Vecchio, D., Huang, Y.-Y., Gupta, A., and Hamblin, M.R. 2013. Light based anti-infectives: ultraviolet C irradiation, photodynamic therapy, blue light, and beyond. Current Opinion in Pharmacology 13:731-762.

12. Wang, Y., Wang, Y., Wang, Y., Murray, C.K., Hamblin, M.R., Hooper, D.C., and Dai, T. 2017. Antimicrobial blue light inactivation of pathogenic microbes: State of the art. Drug resistance updates : reviews and commentaries in antimicrobial and anticancer chemotherapy 33-35:1-22.

13. Dai, T., and Hamblin, M.R. 2017. Visible Blue Light is Capable of Inactivating Candida albicans and Other Fungal Species. Photomedicine and laser surgery 35:345-346.

14. Zhang, Y., Zhu, Y., Chen, J., Wang, Y., Sherwood, M.E., Murray, C.K., Vrahas, M.S., Hooper, D.C., Hamblin, M.R., and Dai, T. 2016. Antimicrobial blue light inactivation of Candida albicans: In vitro and in vivo studies. Virulence 7:536-545.

15. S., G., M., M., G., A.J., J., M.S., D., M.R.M., and H., G.M. 2015. Inactivation of microorganisms isolated from infected lower limb arthroplasties using high-intensity narrow-spectrum (HINS) light. The Bone \& Joint Journal 97-B:283-288.

16. Rosa, L.P., da Silva, F.C., Viana, M.S., and Meira, G.A. 2016. In vitro effectiveness of 455-nm blue LED to reduce the load of Staphylococcus aureus and Candida albicans biofilms in compact bone tissue. Lasers in Medical Science 31:27-32.

17. Ferrer-Espada, R., Liu, X., Goh, X.S., and Dai, T. 2019. Antimicrobial Blue Light Inactivation of Polymicrobial Biofilms. Frontiers in Microbiology 10.

18. 2016. Comparative Sensitivity of Trichophyton and Aspergillus Conidia to Inactivation by Violet-Blue Light Exposure. Photomedicine and laser surgery 34:36-41. 
19. Murdoch, L.E., McKenzie, K., Maclean, M., MacGregor, S.J., and Anderson, J.G. 2013. Lethal effects of high-intensity violet 405-nm light on Saccharomyces cerevisiae, Candida albicans, and on dormant and germinating spores of Aspergillus niger. Fungal Biology 117:519-527.

20. Liang, J.-Y., Cheng, C.-W., Yu, C.-H., and Chen, L.-Y. 2015. Investigations of blue light-induced reactive oxygen species from flavin mononucleotide on inactivation of E. coli. Journal of Photochemistry and Photobiology B: Biology 143:82-88.

21. Kim, M.-J., and Yuk, H.-G. 2017. Antibacterial Mechanism of 405-Nanometer Light-Emitting Diode against Salmonella at Refrigeration Temperature. Applied and environmental microbiology 83:e02582-02516.

22. Keshishyan, E.S., Zaporozhtseva, Z.V., Zenina, O.M., and Zrodnikov, V.S. 2015. Photodynamic Inactivation of Bacteria In Vitro Under the Effect of Blue Light. Bulletin of Experimental Biology and Medicine 158:475-477.

23. Mitchell, R.L., and Anderson, I.C. 1965. Catalase Photoinactivation. Science 150:74-74.

24. Jaishankar, J., and Srivastava, P. 2017. Molecular Basis of Stationary Phase Survival and Applications. Frontiers in Microbiology 8.

25. Ueda, M., Kinoshita, H., Yoshida, T., Kamasawa, N., Osumi, M., and Tanaka, A. 2003. Effect of catalase-specific inhibitor 3-amino-1,2,4-triazole on yeast peroxisomal catalase in vivo. FEMS Microbiology Letters 219:93-98.

26. Meletiadis, J., Verweij, P.E., Te Dorsthorst, D.T.A., Meis, J.F.G.M., and Mouton, J.W. 2005. Assessing in vitro combinations of antifungal drugs against yeasts and filamentous fungi: comparison of different drug interaction models. Medical Mycology 43:133-152.

27. Odds, F.C. 2003. Synergy, antagonism, and what the chequerboard puts between them. Journal of Antimicrobial Chemotherapy 52:1-1.

28. Boncler, M., Różalski, M., Krajewska, U., Podsędek, A., and Watala, C. 2014. Comparison of PrestoBlue and MTT assays of cellular viability in the assessment of anti-proliferative effects of plant extracts on human endothelial cells. Journal of Pharmacological and Toxicological Methods 69:9-16.

29. Fidel, P.L., Vazquez, J.A., and Sobel, J.D. 1999. $<\mathrm{i}>$ Candida glabrata $</ \mathrm{i}>$ : Review of Epidemiology, Pathogenesis, and Clinical Disease with Comparison to $<\mathrm{i}>\mathrm{C}$. albicans $</ \mathrm{i}>$. Clinical Microbiology Reviews 12:80-96.

30. Trofa, D., Gácser, A., and Nosanchuk, J.D. 2008. Candida parapsilosis, an emerging fungal pathogen. Clinical Microbiology Reviews 21:606-625.

31. Hawkins, J.L., and Baddour, L.M. 2003. Candida lusitaniae Infections in the Era of Fluconazole Availability. Clinical Infectious Diseases 36:e14-e18.

32. Demers, E.G., Biermann, A.R., Masonjones, S., Crocker, A.W., Ashare, A., Stajich, J.E., and Hogan, D.A. 2018. Evolution of drug resistance in an antifungal-naive chronic <em>Candida lusitaniae</em> infection. Proceedings of the National Academy of Sciences 115:12040-12045.

33. Ramos, L.S., Figueiredo-Carvalho, M.H.G., Barbedo, L.S., Ziccardi, M., Chaves, A.L.S., Zancopé-Oliveira, R.M., Pinto, M.R., Sgarbi, D.B.G., Dornelas-Ribeiro, M., Branquinha, M.H., et al. 2014. Candida haemulonii complex: species identification and antifungal susceptibility profiles of clinical isolates from Brazil. Journal of Antimicrobial Chemotherapy 70:111-115.

34. Delattin, N., Cammue, B.P., and Thevissen, K. 2014. Reactive oxygen species-inducing antifungal agents and their activity against fungal biofilms. Future Medicinal Chemistry 6:77-90.

35. Mesa-Arango, A.C., Trevijano-Contador, N., Román, E., Sánchez-Fresneda, R., Casas, C., Herrero, E., Argüelles, J.C., Pla, J., Cuenca-Estrella, M., and Zaragoza, O. 2014. The Production of Reactive Oxygen Species Is a Universal Action Mechanism of Amphotericin B against Pathogenic Yeasts and Contributes to the Fungicidal Effect of This Drug. Antimicrobial agents and chemotherapy 58:6627-6638.

36. Kobayashi, D., Kondo, K., Uehara, N., Otokozawa, S., Tsuji, N., Yagihashi, A., and Watanabe, N. 2002. Endogenous Reactive Oxygen Species Is an Important Mediator of Miconazole Antifungal Effect. Antimicrobial agents and chemotherapy 46:3113-3117. 
37. Aguirre, J., Hansberg, W., and Navarro, R. 2006. Fungal responses to reactive oxygen species. Medical Mycology 44:S101-S107.

38. Linares, C.E.B., Giacomelli, S.R., Altenhofen, D., Alves, S.H., Morsch, V.M., and Schetinger, M.R.C. 2013. Fluconazole and amphotericin-B resistance are associated with increased catalase and superoxide dismutase activity in Candida albicans and Candida dubliniensis. Revista da Sociedade Brasileira de Medicina Tropical 46:752-758.

39. Satoh, K., Makimura, K., Hasumi, Y., Nishiyama, Y., Uchida, K., and Yamaguchi, H. 2009. Candida auris sp. nov., a novel ascomycetous yeast isolated from the external ear canal of an inpatient in a Japanese hospital. Microbiology and Immunology 53:41-44.

40. Rhodes, J., and Fisher, M.C. 2019. Global epidemiology of emerging Candida auris. Current Opinion in Microbiology 52:84-89.

41. Prestel, C., Anderson, E., Forsberg, K., Lyman, M., de Perio, M.A., Kuhar, D., Edwards, K., Rivera, M., Shugart, A., Walters, M., et al. 2021. Candida auris Outbreak in a COVID-19 Specialty Care Unit - Florida, July-August 2020. MMWR. Morbidity and mortality weekly report 70:56-57.

42. Pathirana, R.U., Friedman, J., Norris, H.L., Salvatori, O., McCall, A.D., Kay, J., and Edgerton, M. 2018. Fluconazole-Resistant Candida auris Is Susceptible to Salivary Histatin 5 Killing and to Intrinsic Host Defenses. Antimicrobial agents and chemotherapy 62:e1872-01817.

43. Cortegiani, A., Misseri, G., Fasciana, T., Giammanco, A., Giarratano, A., and Chowdhary, A. 2018. Epidemiology, clinical characteristics, resistance, and treatment of infections by Candida auris. Journal of Intensive Care 6:69.

44. Ku, T.S.N., Walraven, C.J., and Lee, S.A. 2018. Candida auris: Disinfectants and Implications for Infection Control. Frontiers in Microbiology 9:726-726.

45. Gilbert, A.S., Wheeler, R.T., and May, R.C. 2014. Fungal Pathogens: Survival and Replication within Macrophages. Cold Spring Harbor perspectives in medicine 5:a019661-a019661.

46. Vylkova, S., Carman, A.J., Danhof, H.A., Collette, J.R., Zhou, H., Lorenz, M.C., and Taylor, J.W. 2011. The Fungal Pathogen Candida albicans Autoinduces Hyphal Morphogenesis by Raising Extracellular pH. mBio 2:e0055-00011.

47. Nakagawa, Y. 2008. Catalase gene disruptant of the human pathogenic yeast Candida albicans is defective in hyphal growth, and a catalase-specific inhibitor can suppress hyphal growth of wildtype cells. Microbiology and Immunology 52:16-24.

48. Mayer, F.L., Wilson, D., and Hube, B. 2013. Candida albicans pathogenicity mechanisms. Virulence 4:119-128.

49. Dai, T., Arce, V.J.B.d., Tegos, G.P., and Hamblin, M.R. 2011. Blue Dye and Red Light, a Dynamic Combination for Prophylaxis and Treatment of Cutaneous Candida albicans Infections in Mice. Antimicrobial agents and chemotherapy 55:5710-5717.

50. Yücesoy, M., and Marol, S. 2003. Performance of CHROMAGAR candida and BIGGY agar for identification of yeast species. Annals of clinical microbiology and antimicrobials 2:8-8.

51. Chen, H., Zhou, X., Ren, B., and Cheng, L. 2020. The regulation of hyphae growth in Candida albicans. Virulence 11:337-348.

52. Wiederhold, N.P. 2017. Antifungal resistance: current trends and future strategies to combat. Infection and drug resistance 10:249-259.

53. Fekkar, A., Lampros, A., Mayaux, J., Poignon, C., Demeret, S., Constantin, J.-M., Marcelin, A.G., Monsel, A., Luyt, C.-E., and Blaize, M. 2021. Occurrence of Invasive Pulmonary Fungal Infections in Patients with Severe COVID-19 Admitted to the ICU. American journal of respiratory and critical care medicine 203:307-317.

54. Saravolatz, L.D., Ostrosky-Zeichner, L., Marr, K.A., Rex, J.H., and Cohen, S.H. 2003. Amphotericin B: Time for a New "Gold Standard". Clinical Infectious Diseases 37:415-425.

55. Dong, P.-T., Zong, C., Dagher, Z., Hui, J., Li, J., Zhan, Y., Zhang, M., Mansour, M.K., and Cheng, J.-X. 2021. Polarization-sensitive stimulated Raman scattering imaging resolves amphotericin B orientation in <em >Candida</em> membrane. Science Advances 7:eabd5230. 
56. Fanos, V., and Cataldi, L. 2000. Amphotericin B-Induced Nephrotoxicity: A Review. Journal of Chemotherapy 12:463-470.

57. Huang, X., Hurabielle, C., Drummond, R.A., Bouladoux, N., Desai, J.V., Sim, C.K., Belkaid, Y., Lionakis, M.S., and Segre, J.A. 2021. Murine model of colonization with fungal pathogen Candida auris to explore skin tropism, host risk factors and therapeutic strategies. Cell Host \& Microbe 29:210-221.e216.

58. Johnson, C.J., Kernien, J.F., Hoyer, A.R., and Nett, J.E. 2017. Mechanisms involved in the triggering of neutrophil extracellular traps (NETs) by Candida glabrata during planktonic and biofilm growth. Scientific Reports 7:13065.

59. Dong, P.-T., Jusuf, S., Hui, J., Zhan, Y., Zhu, Y., Liu, G.Y., and Cheng, J.-X. 2021. Photoinactivation of Catalase Sensitizes Wide-Ranging Bacteria to ROS-Producing Agents and Immune Cells. bioRxiv:2021.2006.2024.449847.

60. Dong, P.T., Mohammad, H., Hui, J., Leanse, L.G., Li, J., Liang, L., Dai, T., Seleem, M.N., and Cheng, J.X. 2019. Photolysis of Staphyloxanthin in Methicillin-Resistant Staphylococcus aureus Potentiates Killing by Reactive Oxygen Species. 6:1900030.

61. Hui, J., Dong, P.-T., Liang, L., Mandal, T., Li, J., Ulloa, E.R., Zhan, Y., Jusuf, S., Zong, C., Seleem, M.N., et al. 2020. Photo-Disassembly of Membrane Microdomains Revives Conventional Antibiotics against MRSA. Advanced Science 7:1903117.

62. Jusuf, S., Dong, P.-T., Hui, J., Ulloa, E.R., Liu, G.Y., and Cheng, J.-X. 2021. Granadaene Photobleaching Reduces the Virulence and Increases Antimicrobial Susceptibility of Streptococcus agalactiae. Photochemistry and Photobiology 97:816-825.

63. Jusuf, S., Hui, J., Dong, P.-T., and Cheng, J.-X. 2020. Staphyloxanthin Photolysis Potentiates Low Concentration Silver Nanoparticles in Eradication of Methicillin-Resistant Staphylococcus aureus. The Journal of Physical Chemistry C 124:5321-5330.

64. Morris-Jones, R., Gomez, B.L., Diez, S., Uran, M., Morris-Jones, S.D., Casadevall, A., Nosanchuk, J.D., and Hamilton, A.J. 2005. Synthesis of melanin pigment by Candida albicans in vitro and during infection. Infection and immunity 73:6147-6150.

65. Ash, C., Dubec, M., Donne, K., and Bashford, T. 2017. Effect of wavelength and beam width on penetration in light-tissue interaction using computational methods. Lasers in medical science 32:1909-1918.

66. Wen, S., Zhou, J., Zheng, K., Bednarkiewicz, A., Liu, X., and Jin, D. 2018. Advances in highly doped upconversion nanoparticles. Nature Communications 9:2415. 
Supplementary information for manuscript

\section{Photoinactivation of catalase sensitizes Candida albicans and Candida auris to ROS-producing agents and immune cells}

Pu-Ting Dong, ${ }^{1,2, \#}$, Yuewei Zhan ${ }^{1,2, \#, ~ S e b a s t i a n ~ J u s u f ~}{ }^{1,2, \#}$, Jie Hui ${ }^{1,2, \#}$, Zeina Dagher ${ }^{3,4}$, Michael K. Mansour ${ }^{3,4,{ }^{*}, \text { Ji-Xin Cheng }}{ }^{1,2, *}$

${ }^{1}$ Department of Biomedical Engineering, Boston University, Boston, MA02215, USA.

${ }^{2}$ Photonics Center, Boston University, Boston, MA02215, USA.

${ }^{3}$ Division of Infectious Diseases, Massachusetts General Hospital, Boston, MA02114, USA.

${ }^{4}$ Harvard Medical School, Boston, MA02115, USA.

${ }^{\star}$ Corresponding authors. Emails: jxcheng@bu.edu and mkmansour@partners.org

\#These authors contributed equally: Pu-Ting Dong, Yuewei Zhan, Sebastian Jusuf, Jie Hui.

\section{This file includes:}

Supplementary Figures 1-13 

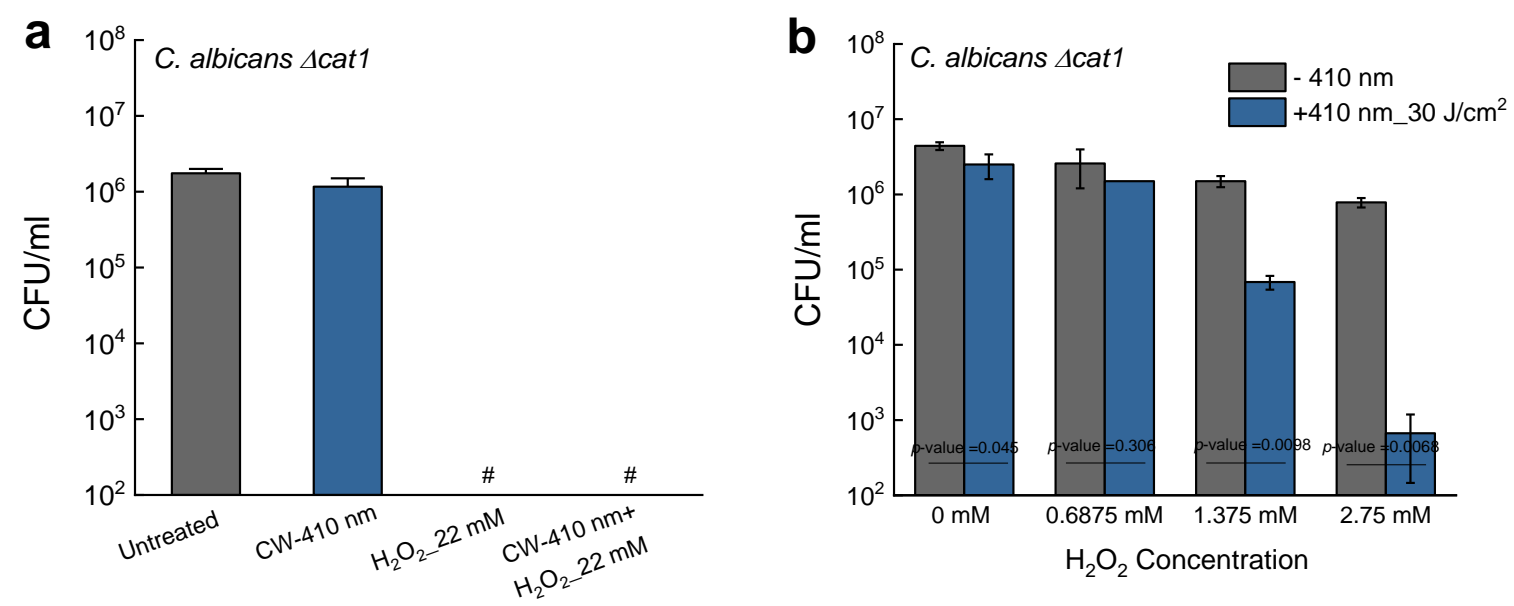

Supplementary Figure 1. CFU/ml of catalase-deficient $C$. albicans $\Delta$ cat1 under different treatment schemes. Data: Mean+SD. N=3. Student unpaired $t$-test. ${ }^{*}: p<0.05,{ }^{* *}: p<0.01 ;{ }^{* *}: p<0.001$.

a

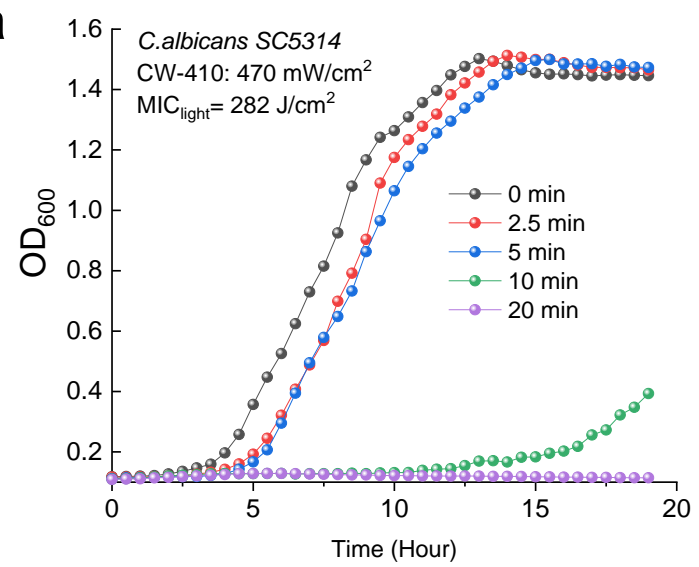

C

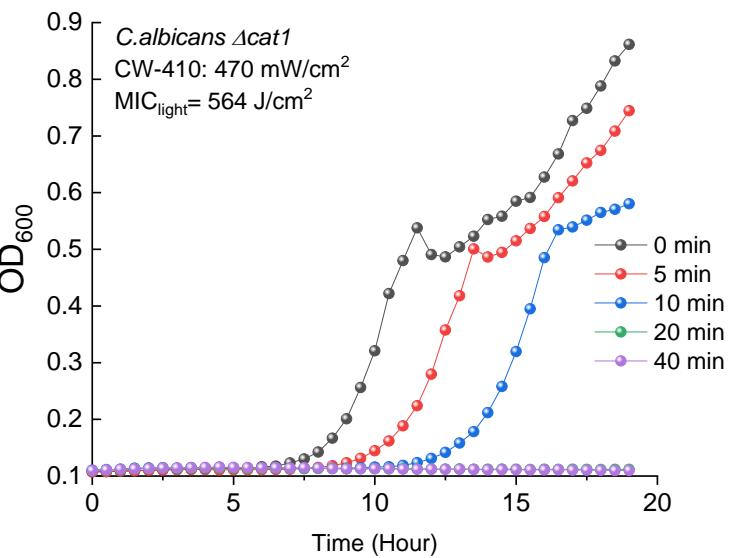

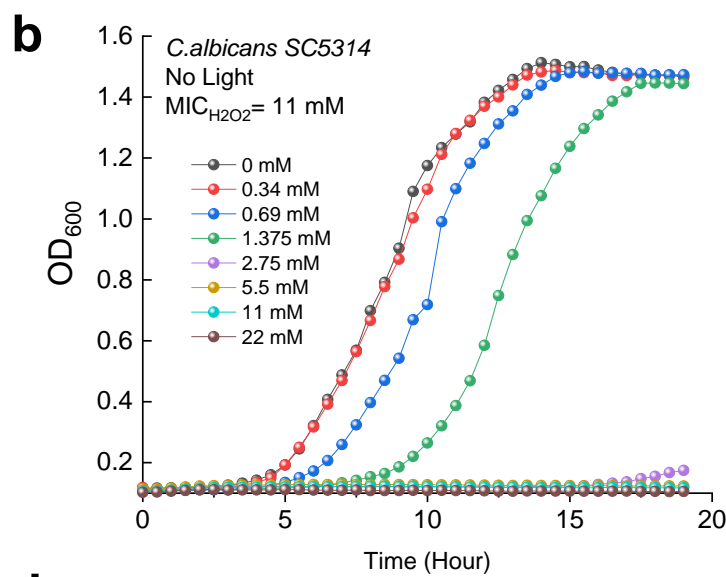

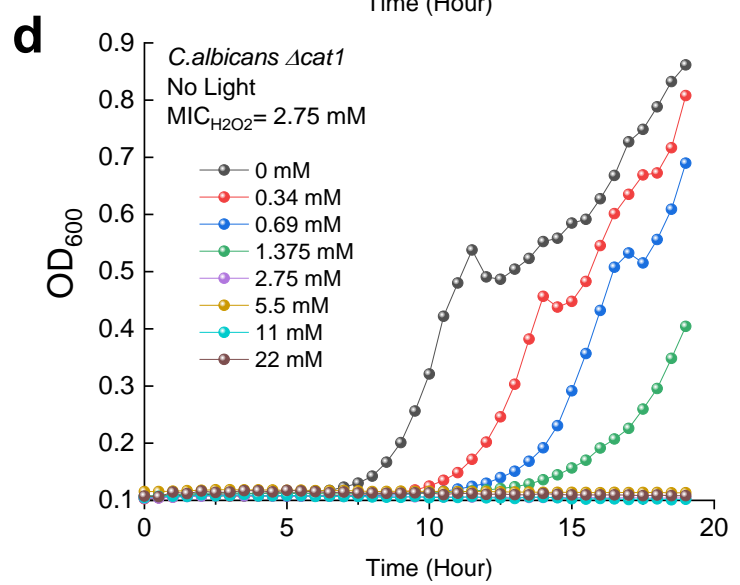

Supplementary Figure 2. Minimal inhibitory concentrations of $410 \mathrm{~nm}$ exposure and $\mathrm{H}_{2} \mathrm{O}_{2}$ against C. albicans SC5314 and catalase-deficient C. albicans Acat1. 

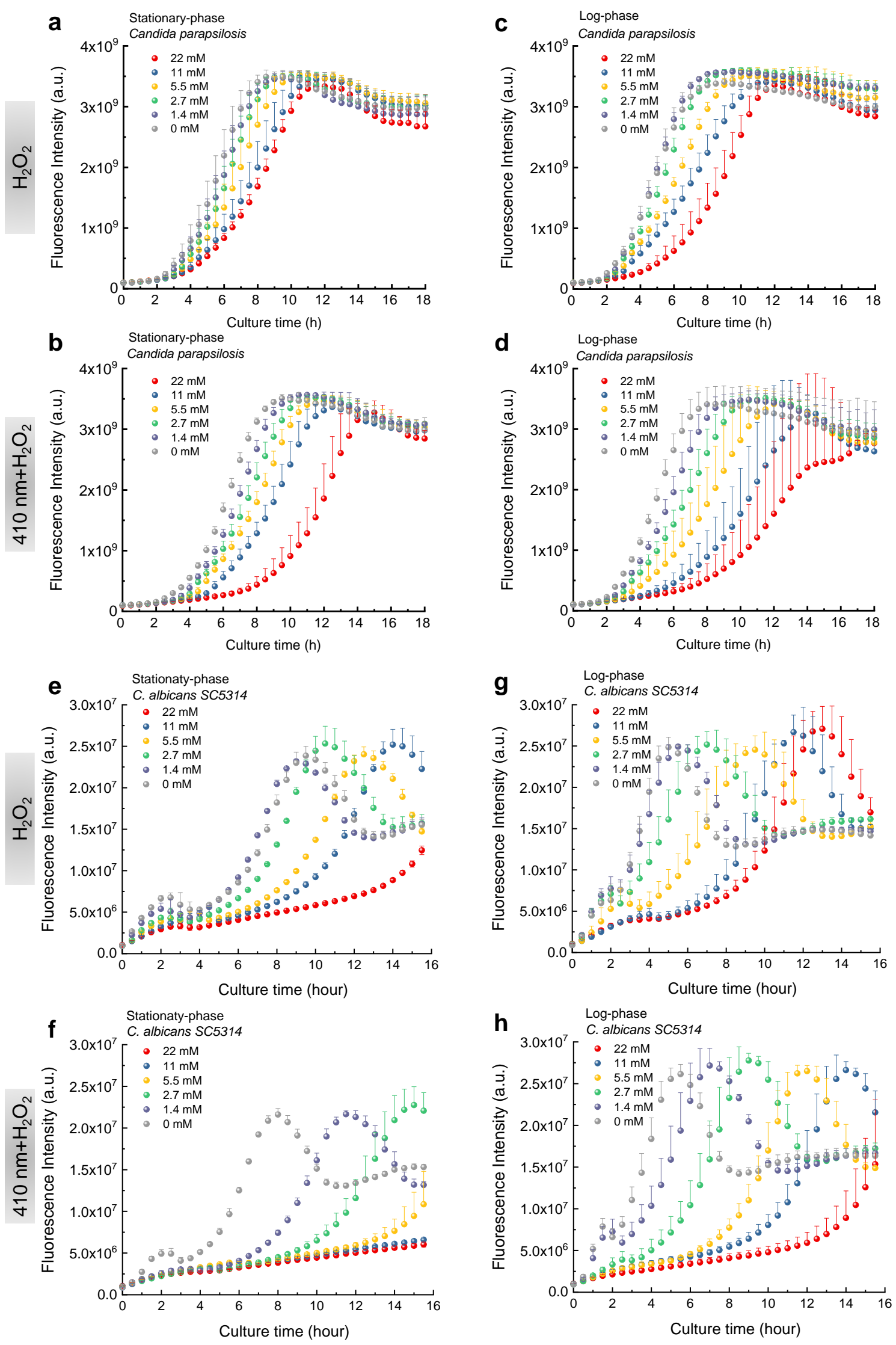


\section{Supplementary Figure 3. Time-course PrestoBlue fluorescence intensity from various fungal species under the treatment of $410 \mathrm{~nm}$ and $\mathrm{H}_{2} \mathrm{O}_{2}$ of various concentrations.}
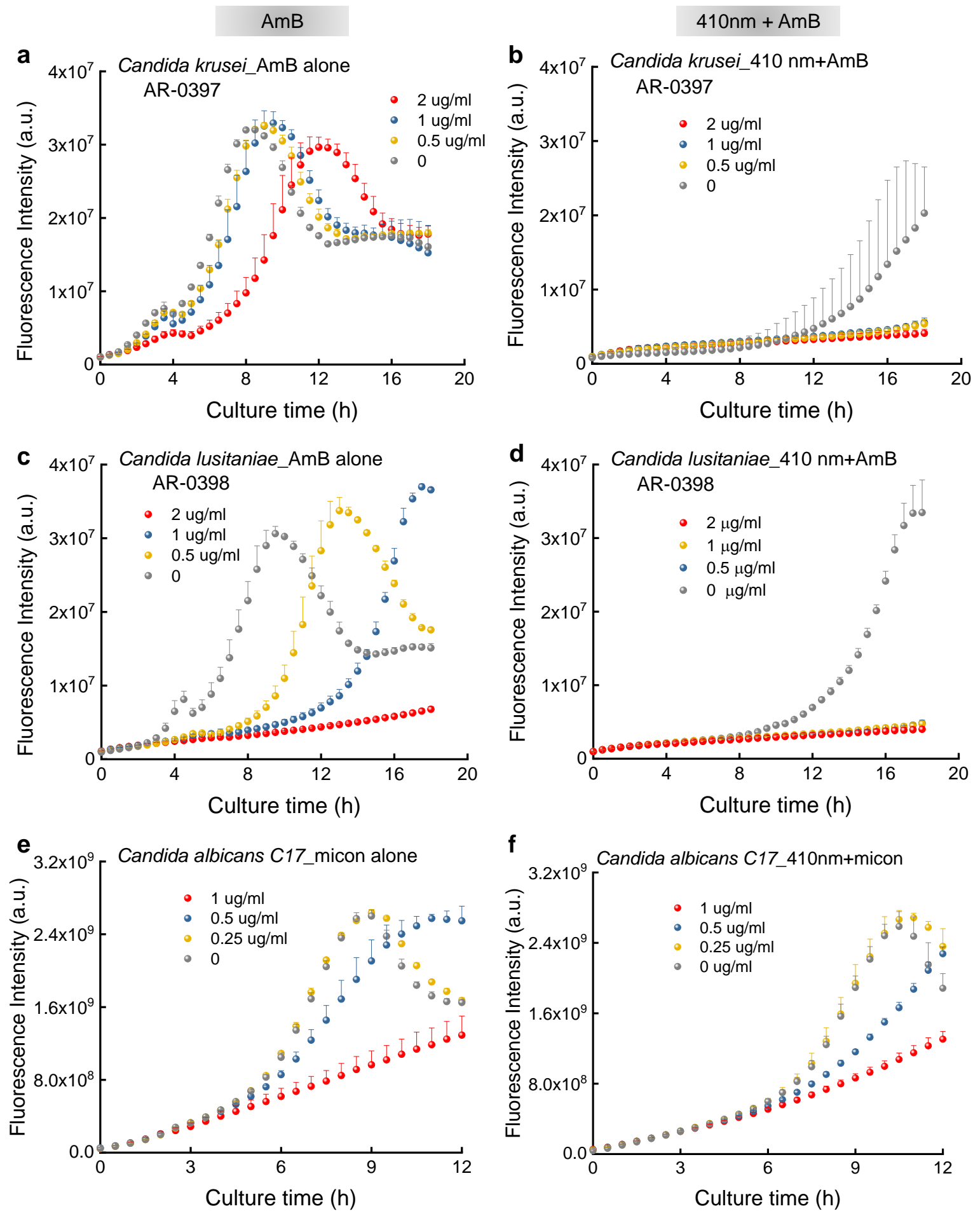

Supplementary Figure 4. Time-course PrestoBlue fluorescence intensity from various fungal species under the treatment of $410 \mathrm{~nm}$ and antifungal drugs. 

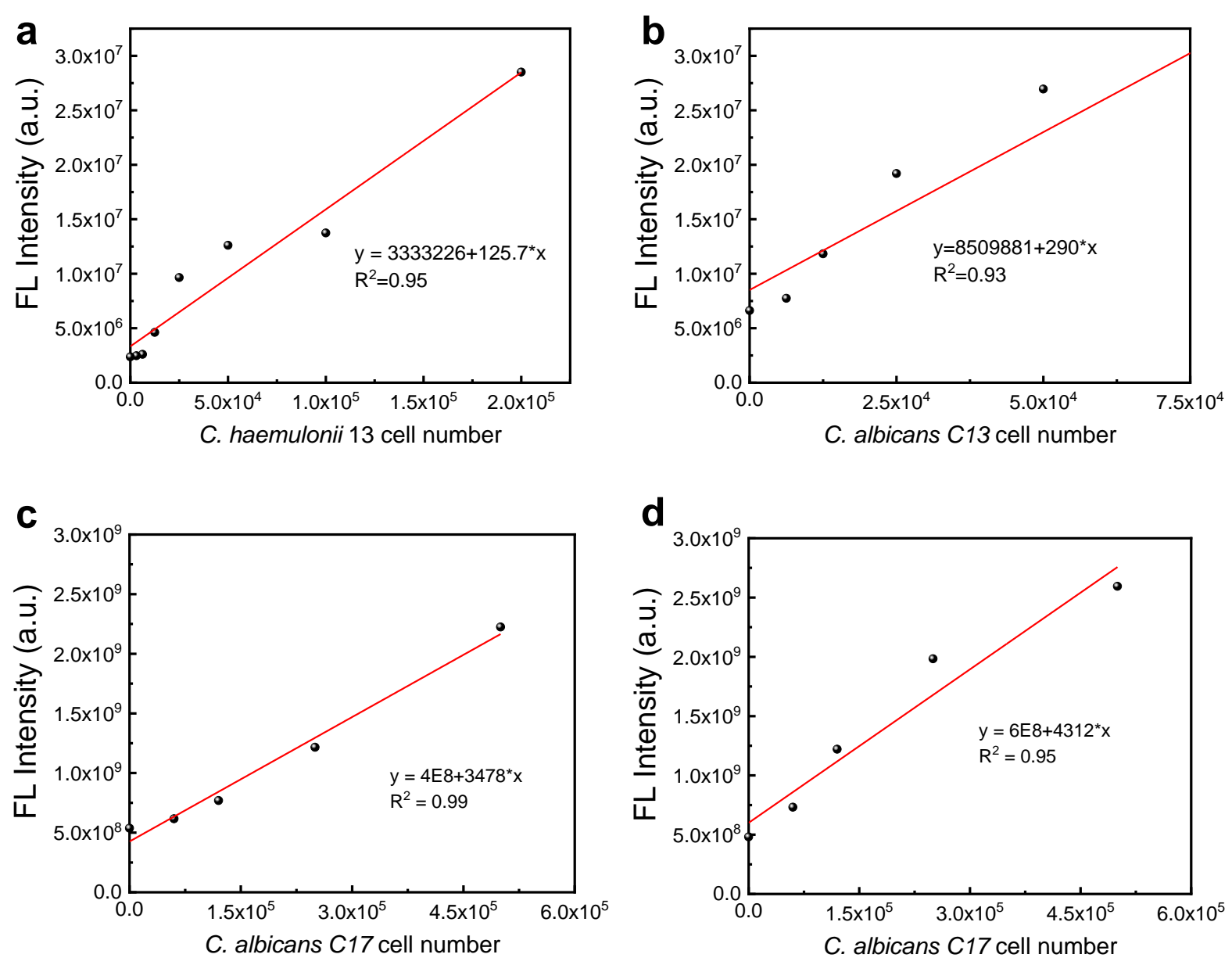

\section{Supplementary Figure 5. Calibration curves of Candida spp. CFU/ml versus PrestoBlue fluorescence intensity.}



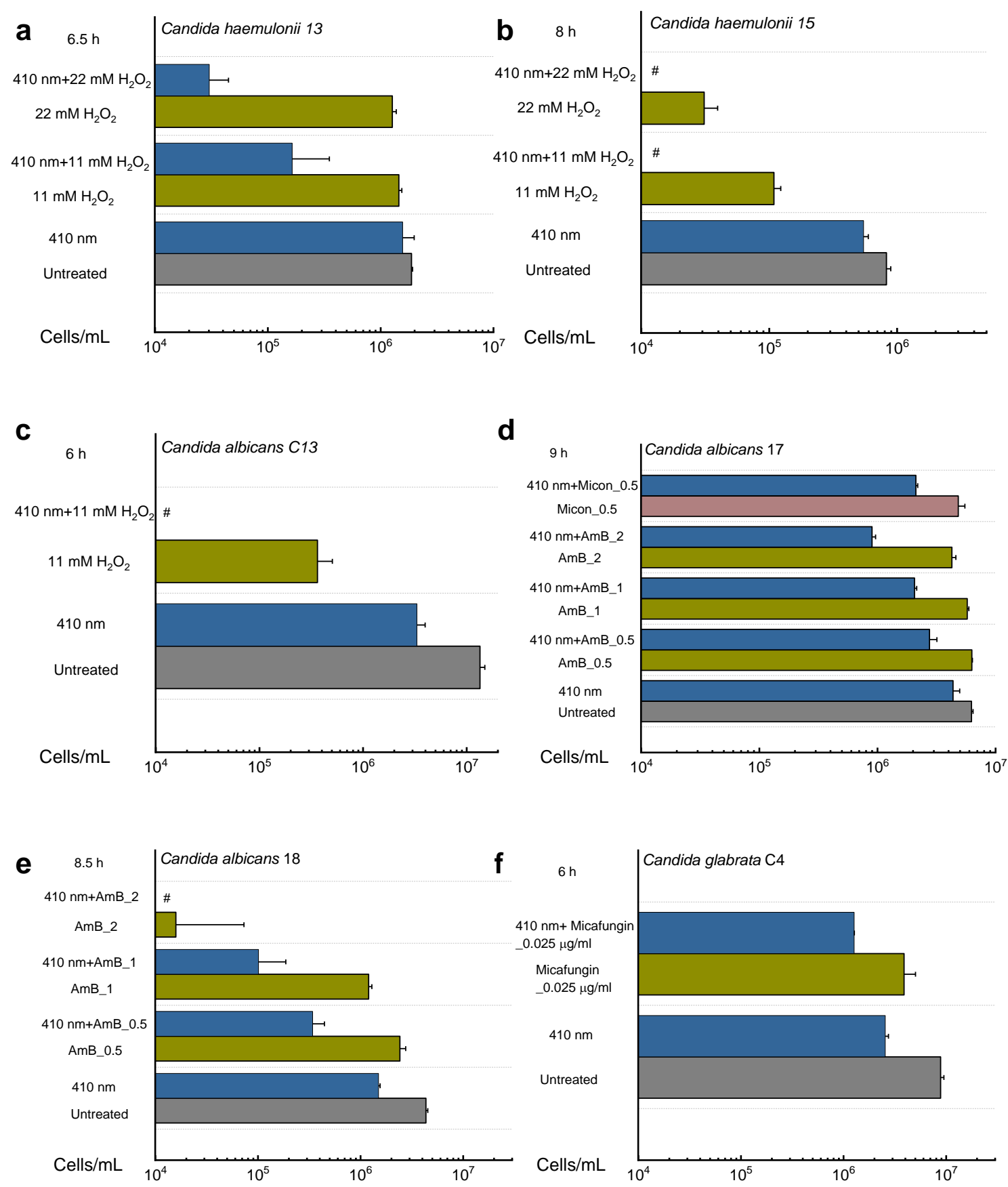

\section{Supplementary Figure 6. Derived Candida cells/mL after different treatments based on the calibration curves. Data: Mean+SD. $\mathrm{N}=3$. Pound sign (\#) means below the detection limit.}




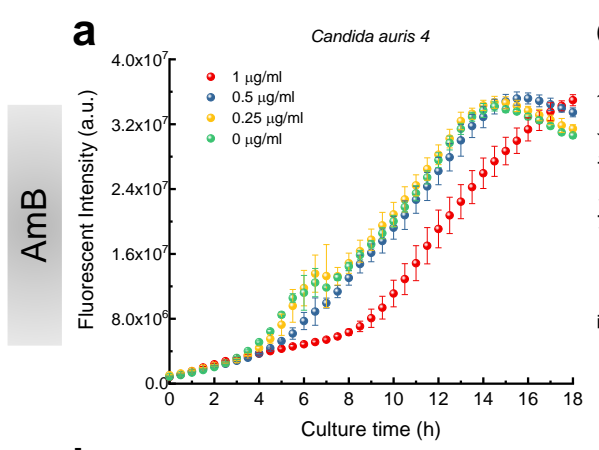

b
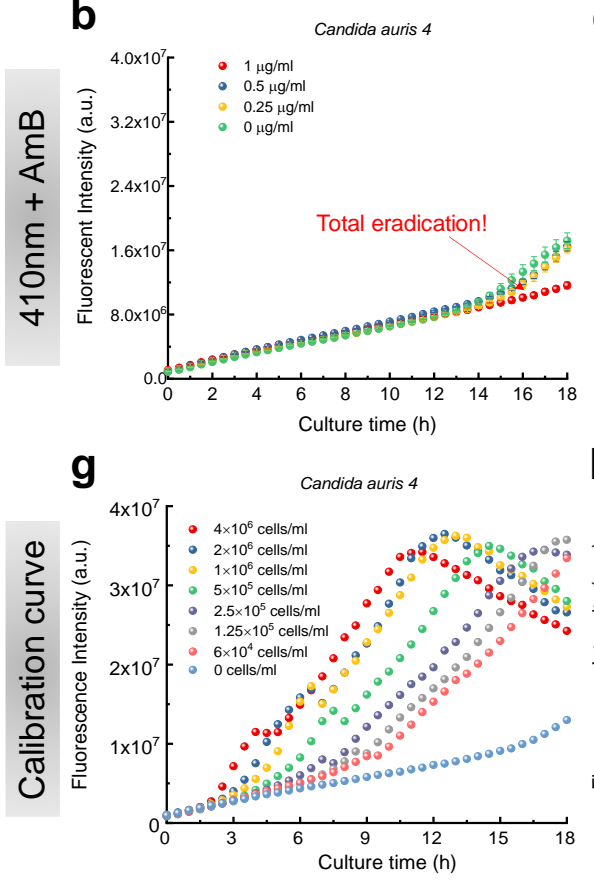

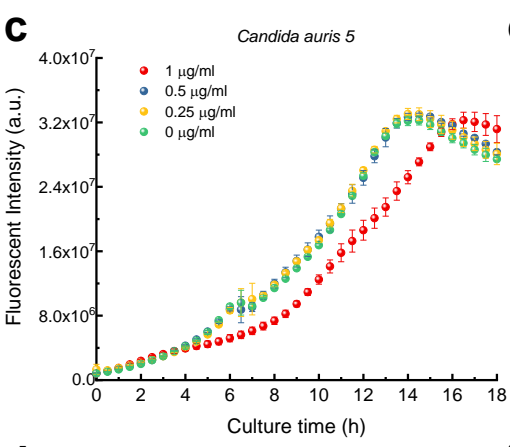

d

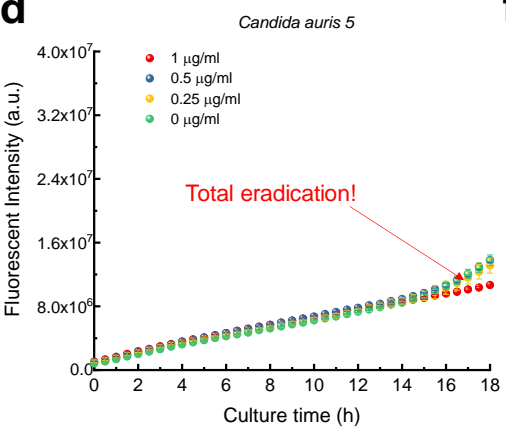

h

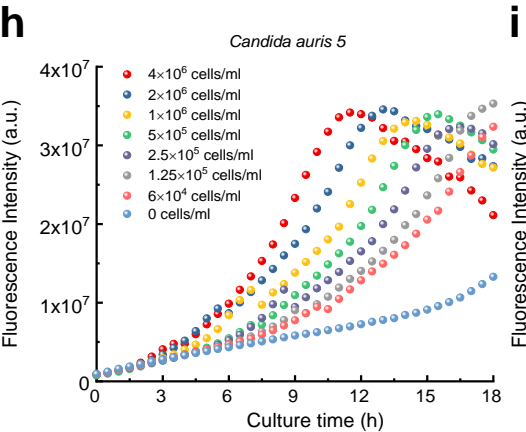

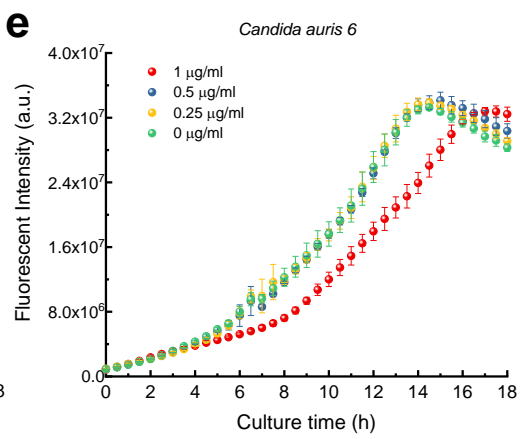

f

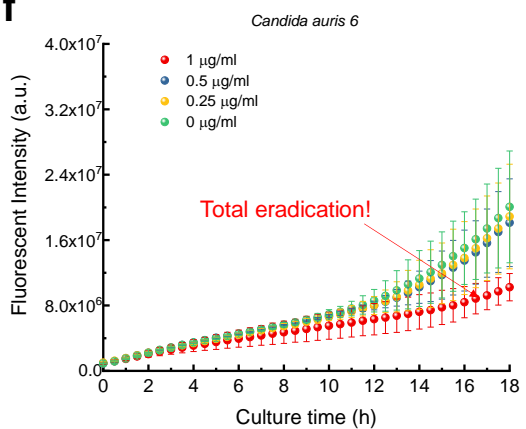

i

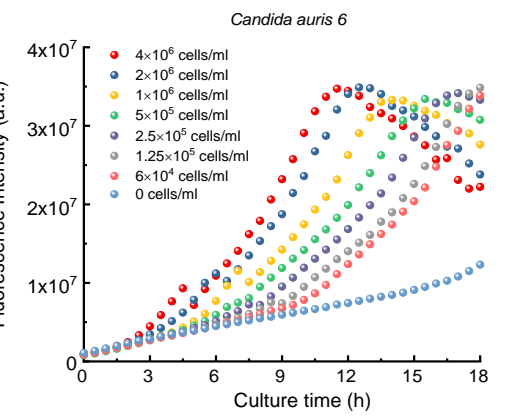

Supplementary Figure 7. Time-course PrestoBlue fluorescence intensity from various Candida auris strains under the treatment of $410 \mathrm{~nm}$ and antifungal drugs. 

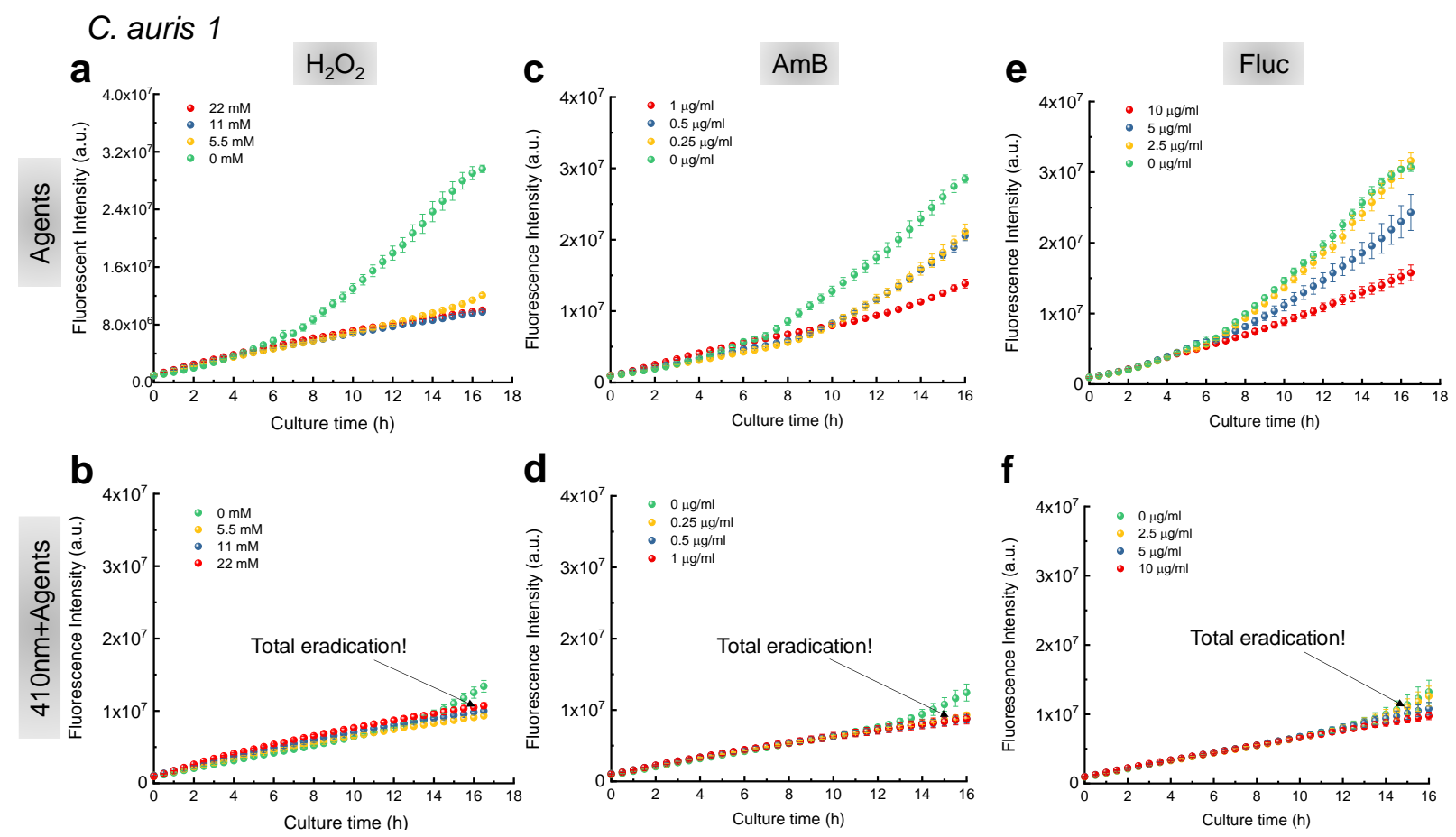

Supplementary Figure 8. Time-course PrestoBlue fluorescence intensity from Candida auris 1 under the treatment of $410 \mathrm{~nm}$ and antifungal agents.

C. auris 2
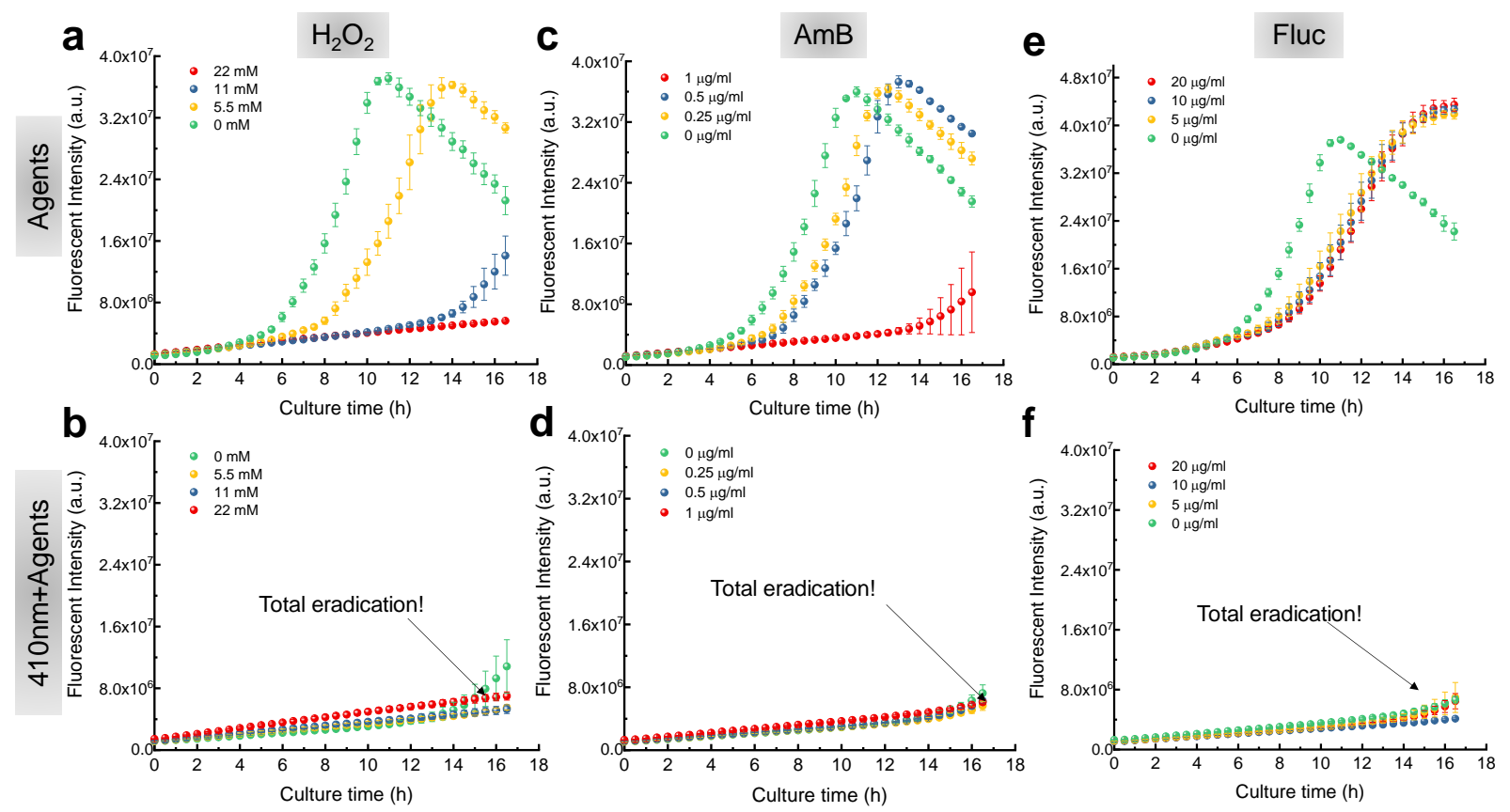

Supplementary Figure 9. Time-course PrestoBlue fluorescence intensity from Candida auris 2 under the treatment of $410 \mathbf{~ n m}$ and antifungal agents. 
C. auris 3
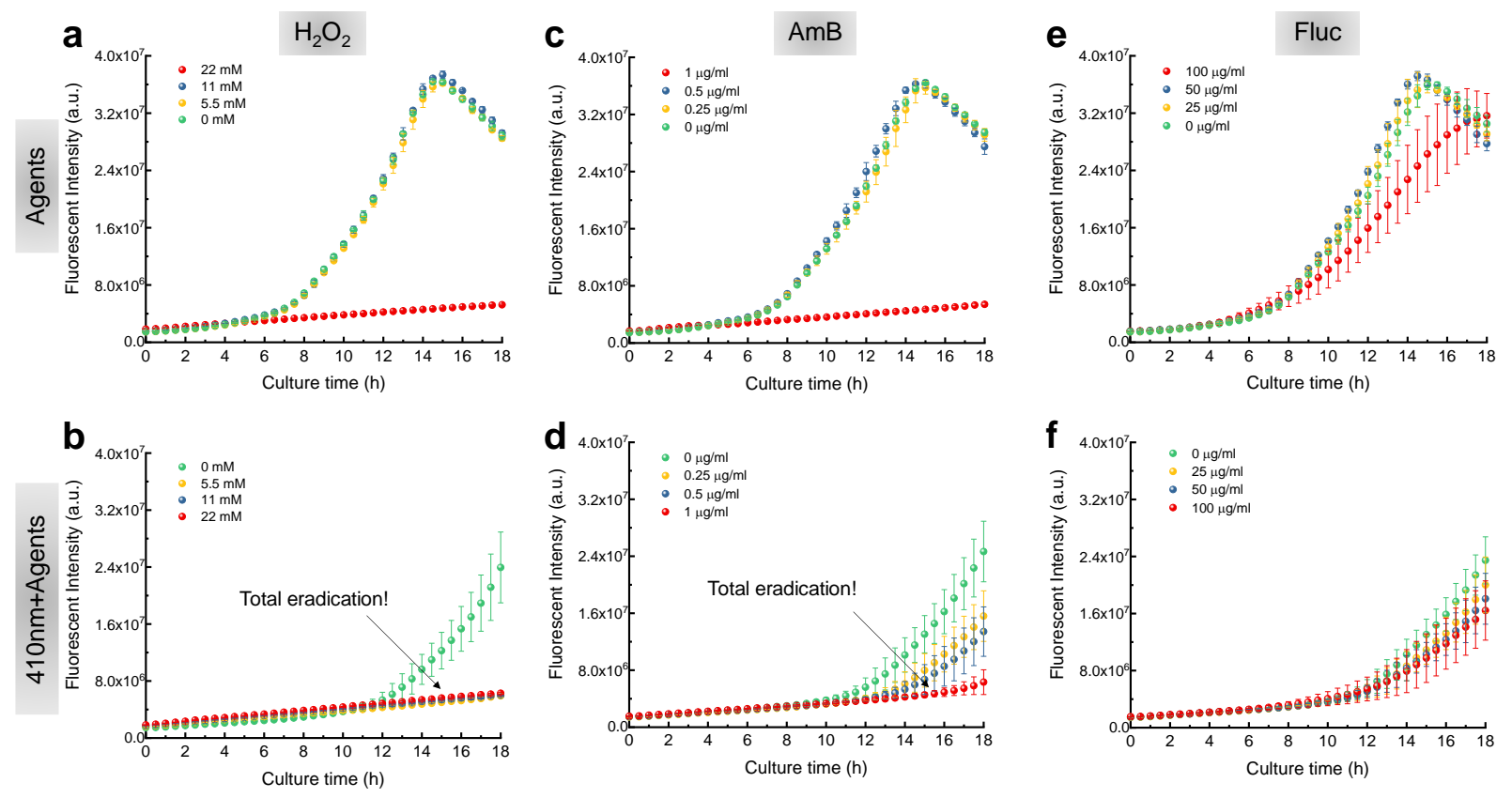

Supplementary Figure 10. Time-course PrestoBlue fluorescence intensity from Candida auris 3 under the treatment of $410 \mathrm{~nm}$ and antifungal agents.

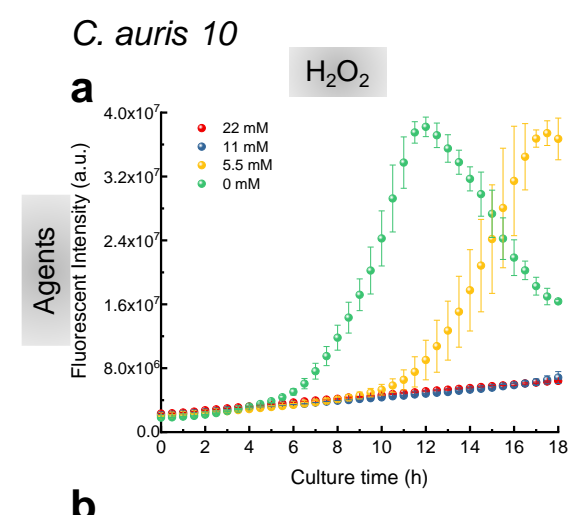

b
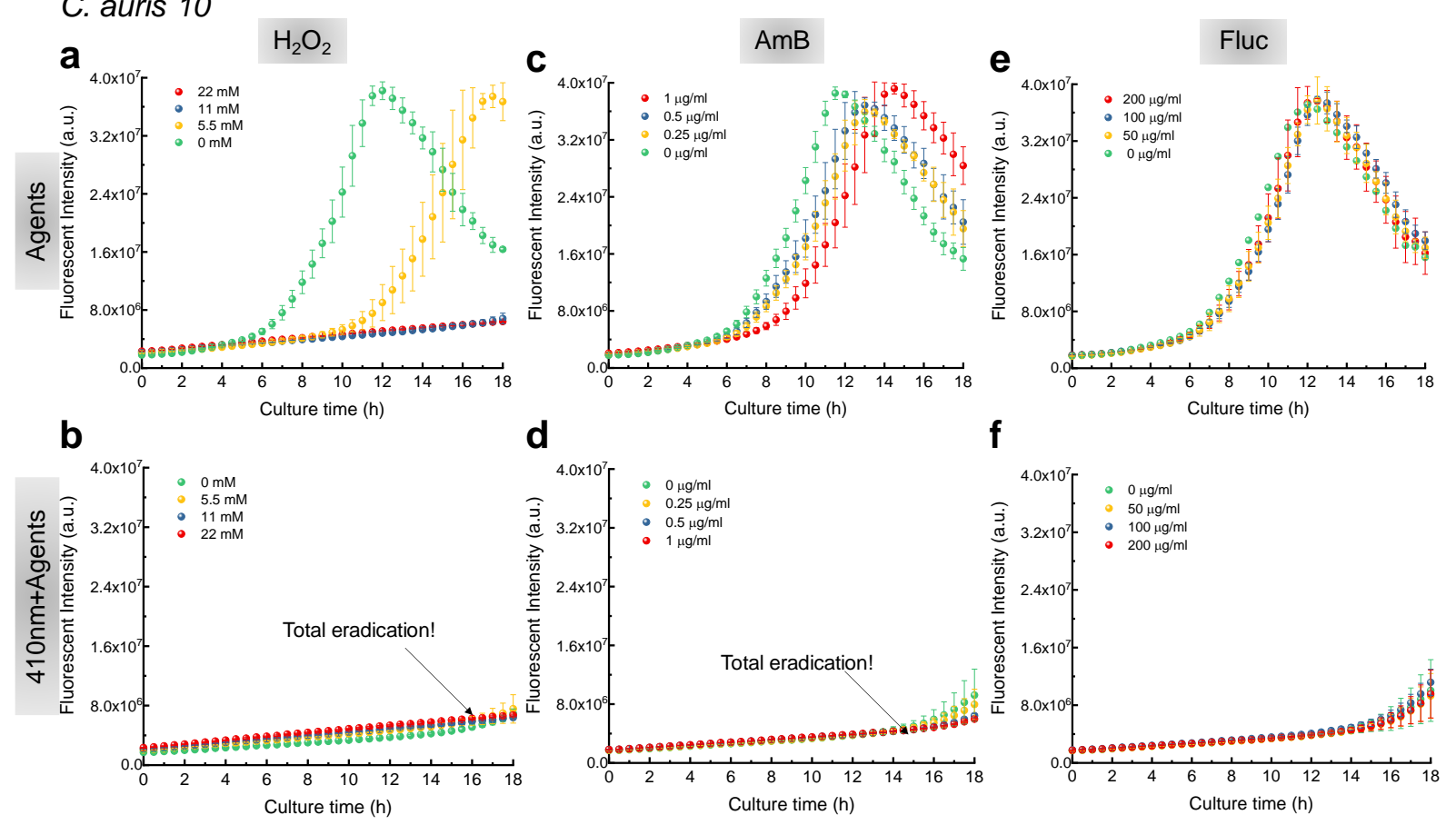

Supplementary Figure 11. Time-course PrestoBlue fluorescence intensity from Candida auris 10 under the treatment of $410 \mathrm{~nm}$ and antifungal agents. 

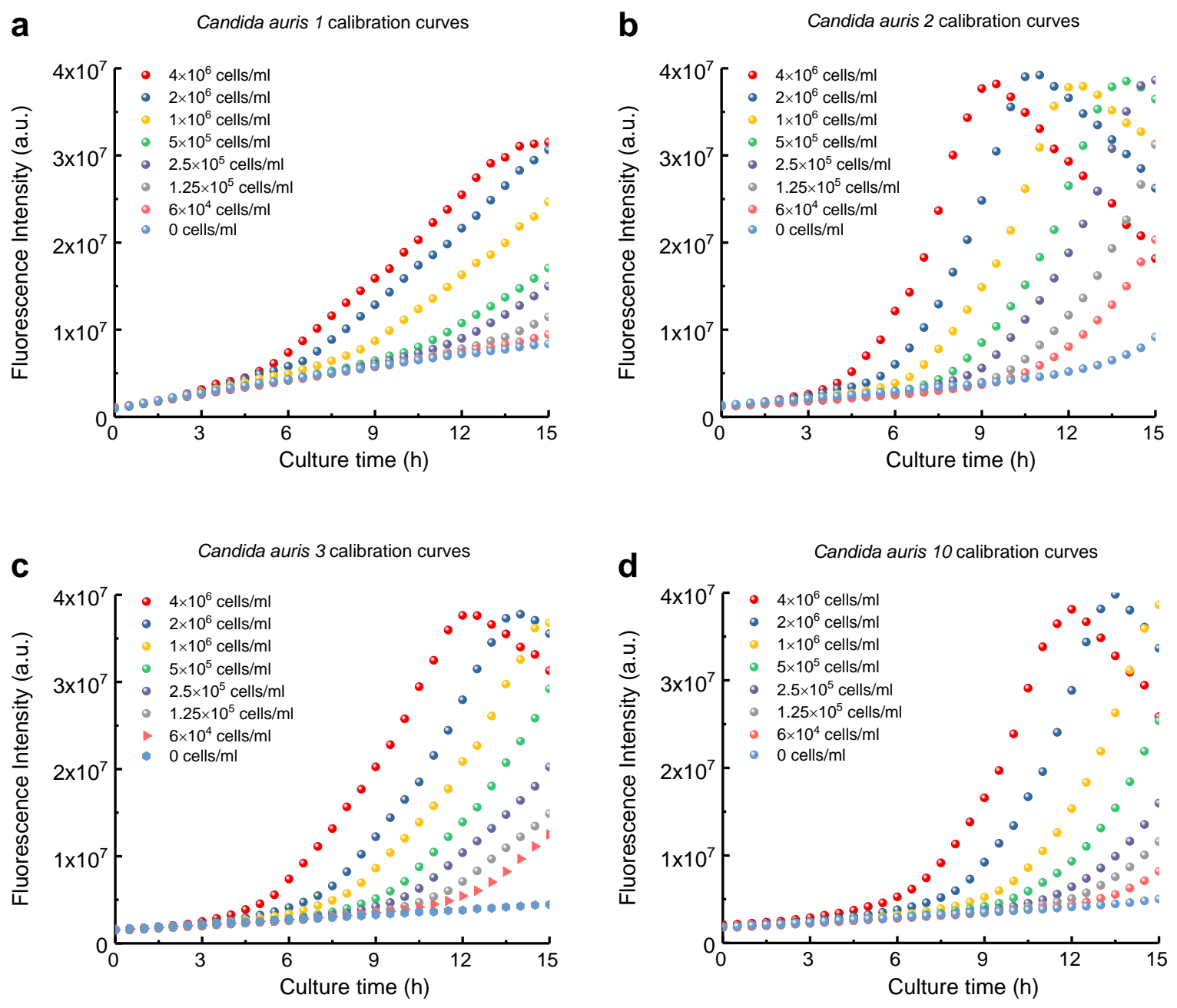

\section{Supplementary Figure 12. Time-course PrestoBlue fluorescence intensity of Candida auris strains at specific concentrations.}



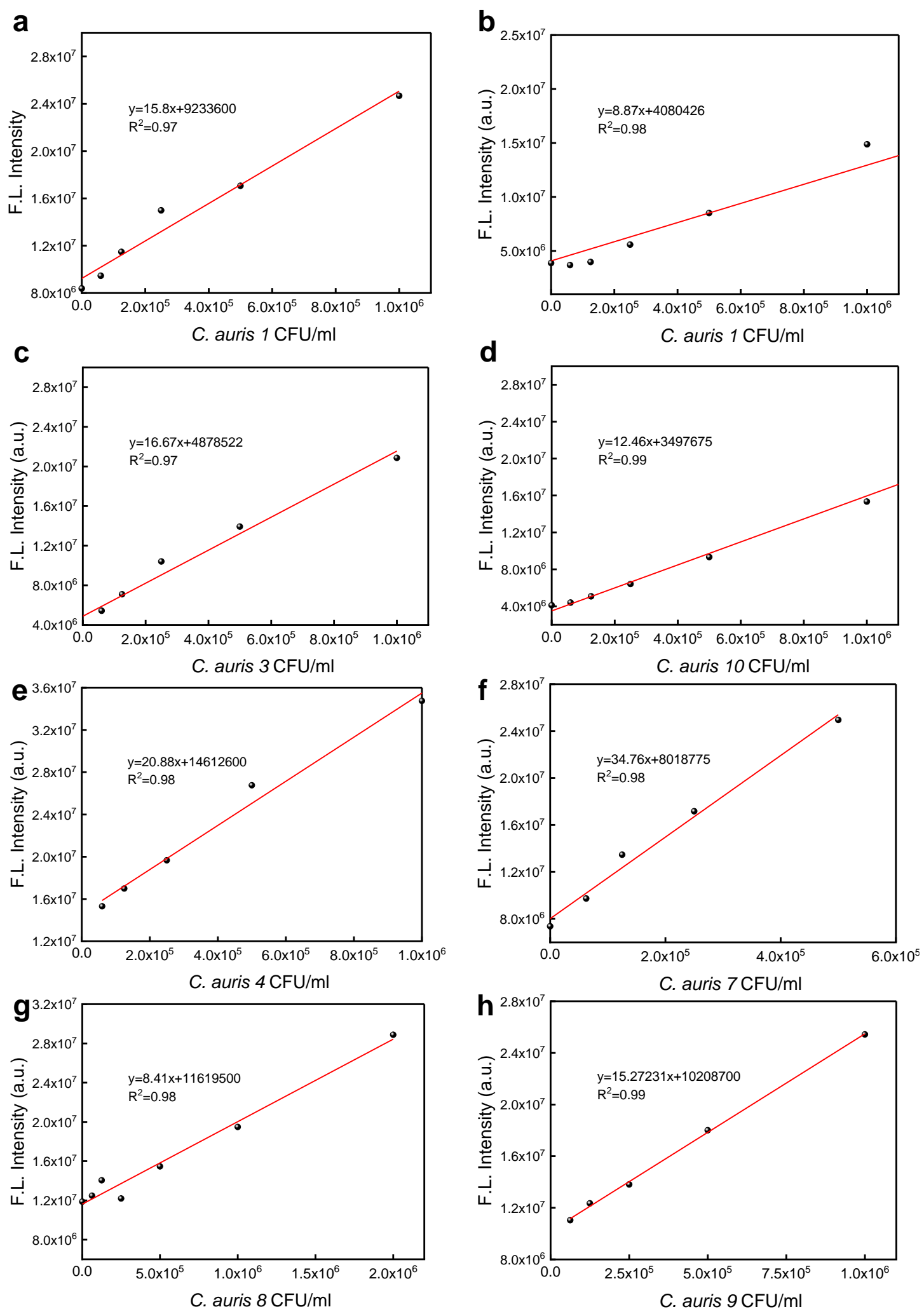

Supplementary Figure 13. Calibration curves of $C$. auris $\mathrm{CFU} / \mathrm{ml}$ versus prestoblue fluorescence intensity. 